A N N A L E S Annales de Bretagne et des Pays de l'Ouest

\title{
Le développement balnéaire breton : une histoire originale
}

Philippe Clairay et Johan Vincent

\section{(2) OpenEdition}

9 Journals

Édition électronique

URL : http://journals.openedition.org/abpo/230

DOI : $10.4000 / a b p o .230$

ISBN : 978-2-7535-1512-3

ISSN : 2108-6443

Éditeur

Presses universitaires de Rennes

Édition imprimée

Date de publication : 31 décembre 2008

Pagination : 201-233

ISBN : 978-2-7535-0808-8

ISSN : 0399-0826

\section{Référence électronique}

Philippe Clairay et Johan Vincent, « Le développement balnéaire breton : une histoire originale »,

Annales de Bretagne et des Pays de l'Ouest [En ligne], 115-4 | 2008, mis en ligne le 31 décembre 2010,

consulté le 21 décembre 2020. URL : http://journals.openedition.org/abpo/230 ; DOI : https://doi.org/ $10.4000 / a b p o .230$ 


\title{
Le développement balnéaire breton : une histoire originale
}

\author{
Philippe ClaIRAY \\ docteur en histoire \\ directeur de l'Écomusée du Moulin de la Sée \\ chercheur correspondant de l'ERHAM - centre André-Chastel, \\ Université Paris IV Sorbonne \\ Johan VINCENT \\ docteur en histoire \\ chargé de recherches à l'Observatoire du patrimoine maritime de Bretagne, \\ GEOMER (UMR 6554) - Université de Bretagne-Occidentale \\ chercheur associé au CERHIO (FRE 3004) - Université de Bretagne-Sud
}

Au regard de la communication actuelle sur la Bretagne touristique, cette région bénéficie de nombreux atouts. La maritimité de la Bretagne est toujours mise en avant, avec ses 2700 kilomètres de côtes (en comptant les îles). Sur le site internet du Comité régional du tourisme de Bretagne, " les anciens villages de pêcheurs côtoient des stations balnéaires réputées. [...] La Bretagne est aussi un pays fier de ses racines, qui a su préserver coutumes et traditions. [...] Surtout, il règne en Bretagne une atmosphère enchantée qui la rend unique, à part ». Pour un touriste friand de pittoresque, un séjour en Bretagne ne peut qu'être bienvenu. Il est loin de la ville et d'une certaine artificialité. La topographie corrobore cette absolue authenticité : le site du Conseil régional de Bretagne proclame que, « bordée par l'Atlantique et la Manche, la Bretagne est bien une terre de marins ${ }^{1}$ ". Ces indications ne sont que le produit simplifié des discours développés depuis plus de deux siècles.

L'activité balnéaire se révèle en fait bien plus complexe, car les acteurs ont dû s'adapter tant aux évolutions nationales du tourisme qu'aux particularismes locaux. Louis Brunet caractérisait en 1963 trois types de stations : celles créées de toutes pièces, celles nées d'un dédoublement de cités préexistantes mais non côtières, et celles, les plus nombreuses, qui

1. Consultation des sites internet le 17 octobre 2007. 
dérivent de ports ou de villages de pêcheurs ${ }^{2}$. Ces considérations générales ne rencontrent-elles pas des variations locales au moment de leur application sur le terrain? Peut-on retrouver des caractéristiques communes à la majorité des stations balnéaires bretonnes? Il s'agit de faire le bilan du phénomène balnéaire en Bretagne. L'observateur contemporain confondra difficilement la station balnéaire bretonne et la station balnéaire des côtes languedociennes ou vendéennes, même s'il existe des points communs. La station balnéaire bretonne apparaît comme modeste, éparpillée, fondée sur un tourisme à taille humaine. Ces spécificités ne sont pas récentes. Pour une partie d'entre elles, elles se retrouvent dans les racines de l'activité balnéaire en Bretagne qui commence au début du XIX ${ }^{\mathrm{e}}$ siècle.

La station balnéaire bretonne d'aujourd'hui est le résultat des images touristiques et des héritages : c'est la première région touristique de France pour les séjours en mer. On peut définir avec Philippe Clairay la station balnéaire comme une agglomération située au bord de la mer, organisée autour d'une ou plusieurs plages, dans laquelle se sont implantés une villégiature de plaisance et des équipements spécifiques, l'ensemble, à destination de loisir, se caractérisant nécessairement par la saisonnalité de son activité économique. Or, historiquement, l'activité balnéaire, que d'autres littoraux connaissent déjà à l'époque, ne s'est implantée que progressivement en Bretagne. Elle est à l'origine d'une profonde mutation des littoraux bretons, tant dans la nouvelle utilisation des rivages que pour l'évolution de l'identité même de la Bretagne.

\section{Le phénomène balnéaire breton : histoire et typologie}

Le tourisme balnéaire, création britannique, se propage en Europe par les fondations de stations allemandes, belges et hollandaises, auxquelles s'ajouta celle de Dieppe, alter ego continentale du Brighton anglais. Suivent les stations du littoral de la Manche à son tour conquis par cette vogue des bains de mer. La Méditerranée, certes déjà fréquentée par les Britanniques, ne répond pas à ces mêmes aspirations pour la balnéation maritime : il s'agit là-bas de trouver une certaine salubrité climatique et d'y passer la saison hivernale, sans qu'il soit pour le moment question de se baigner; les rivages méditerranéens ne seront gagnés par les baigneurs que tardivement, au xx ${ }^{\mathrm{e}}$ siècle. En France, si Boulogne et Dieppe sont nos plus anciennes stations, elles sont dans les décennies 1820-1830 " concurrencées " par de nouvelles inventions, telles Étretat, Arcachon, Granville, Les Sables-d'Olonne, Royan et Pornic. Enfin, entre 1830 et 1848, apparaissent Saint-Malo - Saint-Servan, Le Croisic, Biarritz, Trouville, etc. Après 1850, il devient difficile de détailler toutes les créations de centres de bains de mer, tant elles sont nombreuses. Il est néanmoins intéressant de constater que parmi les toutes premières expérimentations balnéaires se

2. BURNET, Louis, Villégiature et tourisme sur les côtes de France, Paris, Hachette, coll. " Bibliothèque des Guides bleus ", 1963, p. 26-27. 
Le développement balnéaire breton
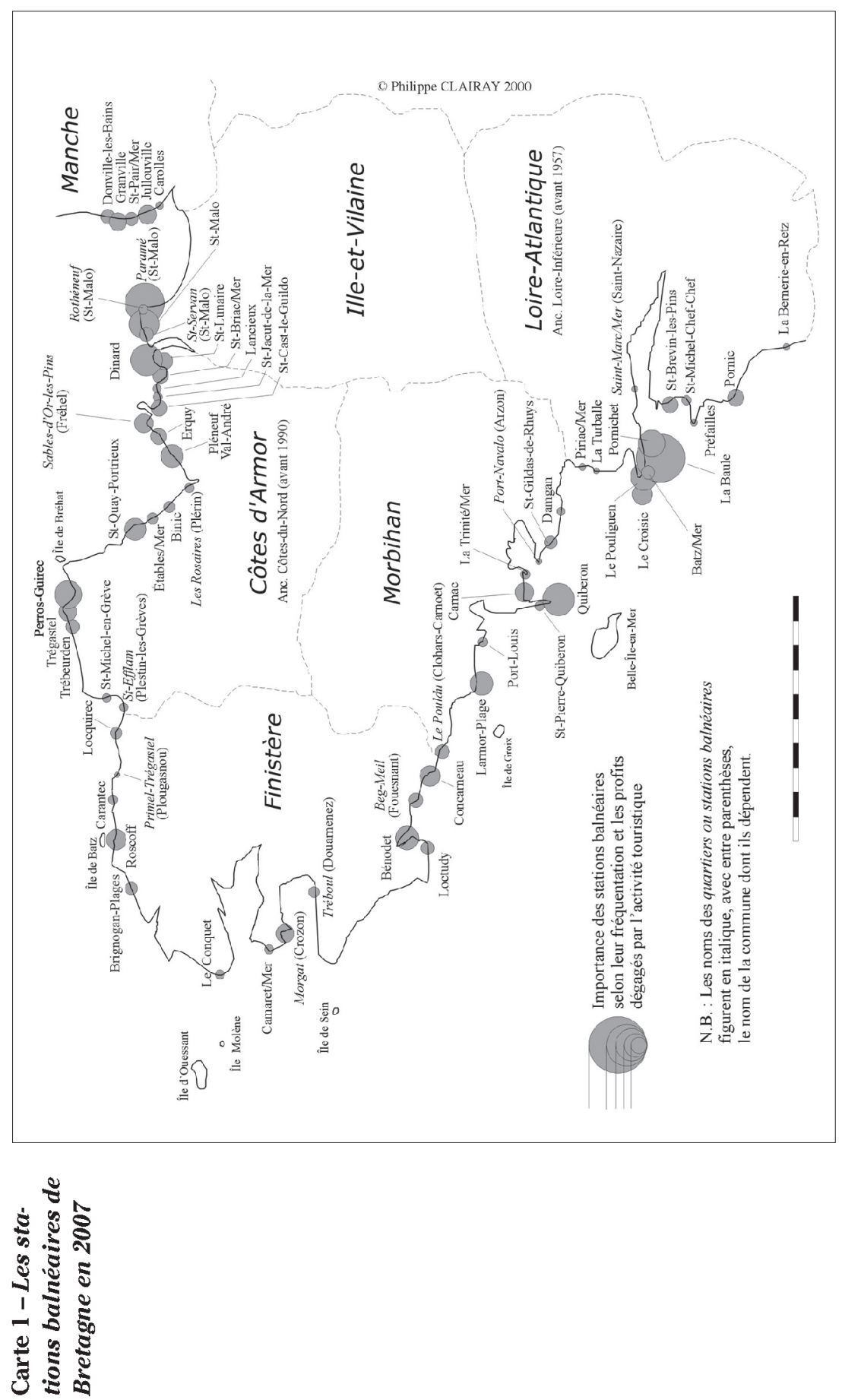
trouvent en bonne place des plages bretonnes, comme le démontre cette frise chronologique.

\section{Figure 1 - Chronologie de l'apparition des stations balnéaires} en Europe, par régions

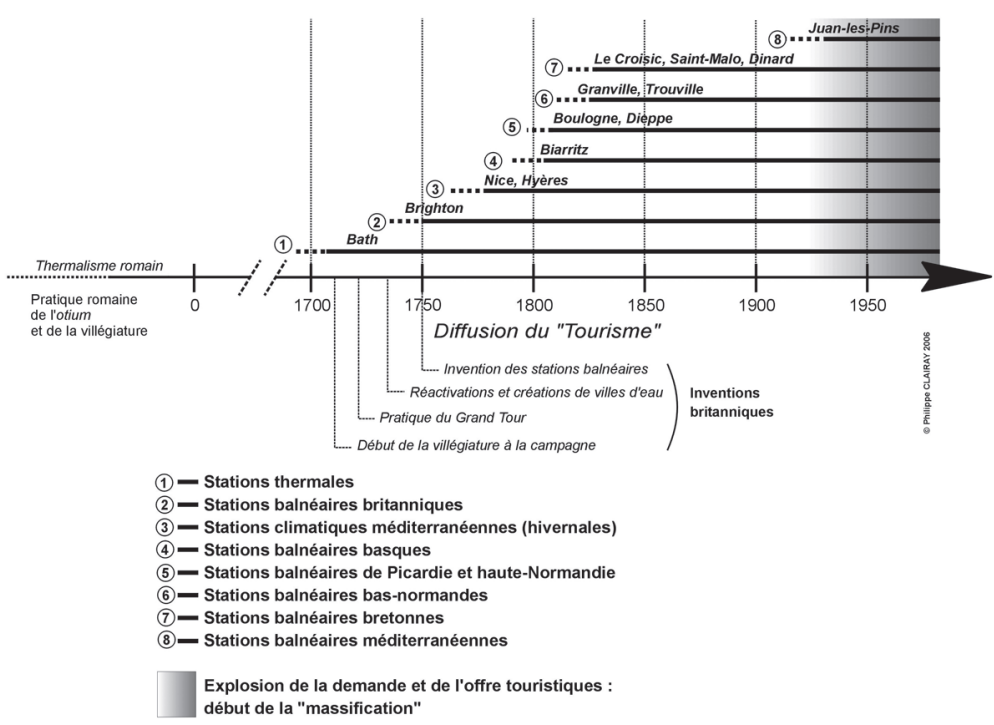

Dans la constellation des stations balnéaires françaises, celles qui naissent sur les côtes de la Manche, mer aux eaux vives, ont une place de choix. Elles ont représenté à l'époque de leur création un nec plus ultra de la balnéation thérapeutique, seule baignade alors pratiquée. La localisation des premiers centres de bains de mer en Bretagne se réalise $a$ priori de manière inintelligible : la lecture d'une carte géographique n'apporte dans ce cas que peu de réponses. Les plus anciennes plages de l'Ouest armoricain aménagées dans le but de pratiquer des bains de mer thérapeutiques sont Granville (fin des années 1820), Saint-Malo (premier établissement de bains de mer en 1835), Pornic (1837) et Le Croisic (1837). Une première observation permet de constater que les deux pôles touristiques majeurs de la Bretagne contemporaine figurent, outre la station normande, au nombre des premières stations balnéaires (l'ensemble malouin et les stations de la presquîile de Guérande).

La Bretagne, par sa diversité littorale, offre un panel de toutes les expériences balnéaires précitées : stations anciennes, aristocratiques, fondées par une ou plusieurs personnalités-leaders; stations plus récentes, fondées ex nihilo; stations qui ont duré et stations qui ont échoué; stations simples et peu équipées; stations très aménagées et au développement complexe; stations orientées dans tous les azimuts, etc. Les questions qui vont être 
Le développement balnéaire breton

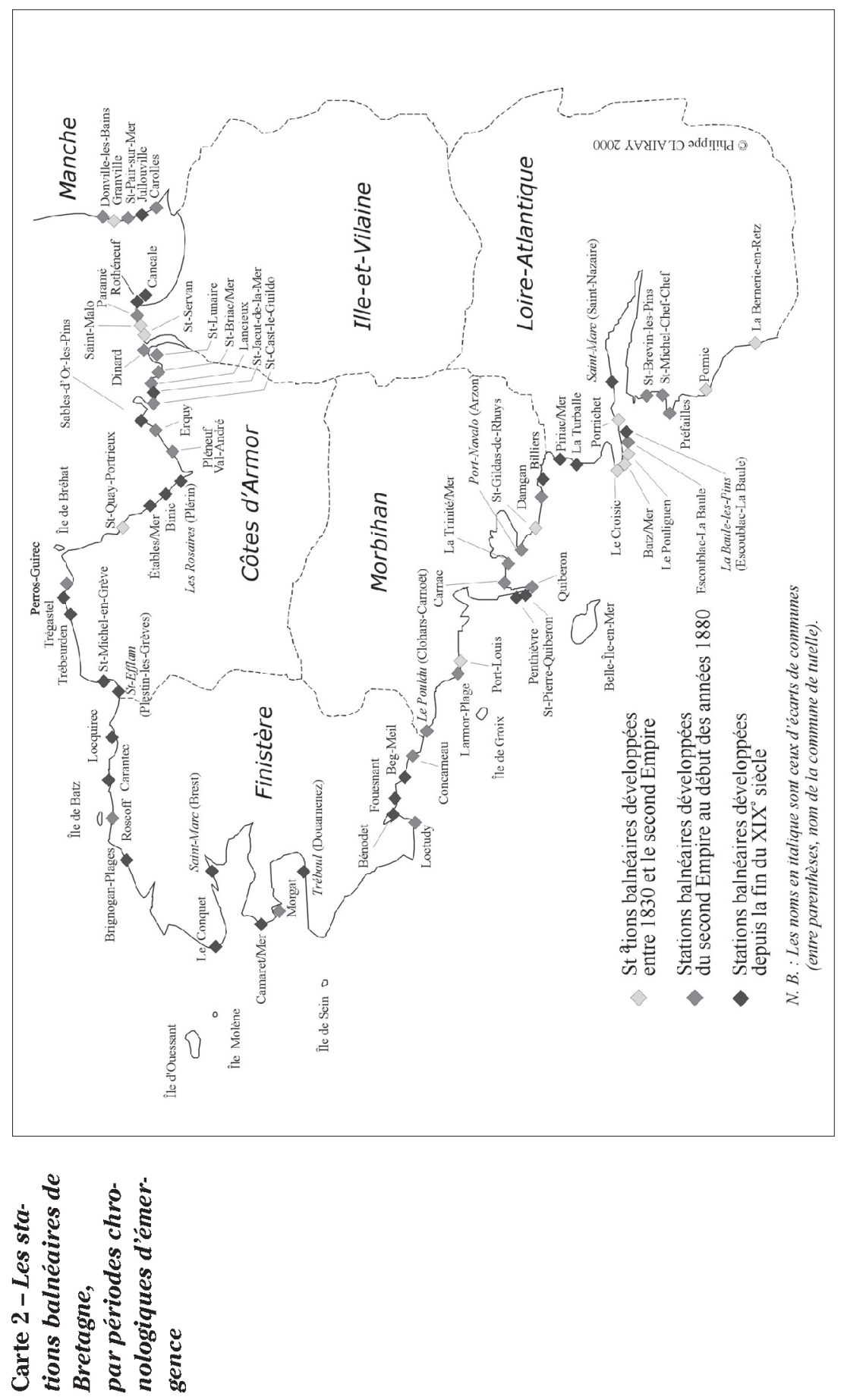


abordées ici visent justement à comprendre pourquoi et comment est née cette multiplicité de stations balnéaires et, si possible, à mettre en évidence des schémas de développement communs.

\section{La localisation des stations balnéaires}

La localisation des stations balnéaires s'accommode mal des réponses géographiques classiques. En Bretagne, les premiers sites occupés sont souvent les sites les plus remarquables, tels l'ensemble malouin (SaintMalo - Dinard) ou celui du Croisic. Mais pourquoi ne pas évoquer comme motivation initiale du " colon ", la beauté des lieux? La subjectivité dans ce choix premier ne doit pas nous effrayer car c'est un critère, pour lors, décisif. Dans nul autre domaine plus que le tourisme cette notion de charme des lieux ne peut être mis de côté tant il semble présider au destin de ces plages. Sans compter que les plages les plus importantes en surface, celles rétrospectivement les plus faciles à aménager, n'ont pas été celles qui ont été développées les premières... Ainsi, sur le littoral breton, de vastes plages inoccupées s'étendent paisiblement à quelques kilomètres de centres balnéaires, eux, très densément peuplés...

Ce goût pour le panorama n'est évidemment pas un fait technique mais le fruit d'un processus culturel qui procède d'une éducation. Il ne touche cependant à son origine qu'une "élite voyageuse ", acculturée au tourisme, et à son corollaire, la pratique de la villégiature. Il n'est donc pas étonnant de trouver des sujets britanniques (les inventeurs du Tour) à l'origine du développement dinardais. Ces personnages sont les gatekeepers, dont le rôle d'" inventeurs " au sens de découvreurs a été bien décrit par Marc Boyer $^{3}$. La station, c'est-à-dire étymologiquement la résidence ${ }^{4}$, va fixer dans le cadre d'une villégiature maritime les baigneurs qui sont aussi des spectateurs de l'océan.

La résidence balnéaire ne semble se fixer dans un premier temps que sur des côtes à relief rocheux et ce, de manière préférentielle par rapport à des côtes basses, sableuses mais dépourvues de promontoires sur lesquels s'installer.

\section{Des origines originales?}

Dans la région du Clos-Poulet, c'est-à-dire les environs de Saint-Malo, émerge de façon assez précoce et inattendue une acculturation hydrominérale. En effet, des sources minérales font l'objet au début du XIX siècle d'une fréquentation importante et des débuts d'une mondanité clanique autour de cercles de buveurs d'eau. Or, il est attesté que ce public, choisi,

3. BOYER, Marc, Histoire du tourisme de masse, Paris, PUF, coll. " Que sais-je? ", 1999, p. 16-22, Ch. II : " L'invention de distinction et la diffusion par imitation. "

4. Les expressions " tourisme balnéaire " et plus encore " station touristique " révèlent une contradiction : le tourisme est un mouvement, la station une immobilité. 
de curistes, jouera plus tard un rôle dans l'émergence des stations balnéaires. Le panégyriste de la Côte d'Émeraude, Eugène Herpin ${ }^{5}$, apporte des éléments parmi les plus intéressants. Ses propos témoignent du passage d'une acculturation liée aux pratiques hydrominérales à la naissance d'une société spécifiquement balnéaire. On ne saurait discuter du caractère essentiel des réflexions " anthropologiques " de cet auteur, réflexions qui nous permettent aussi d'entreprendre une analyse historiographique des premiers bains de mer au Clos-Poulet ${ }^{6}$ : «En 1835, sur la Grand'Grève de Saint-Malo, fut fondé le premier établissement de bains de mer. Le Petit Casino suivit de près, et la belle société de Vaugarni y transporta ses élégances et ses distractions. Dès lors chez nous la mode des eaux minérales est morte. L'ère balnéaire est définitivement ouverte ${ }^{7}$."

L'érudit malouin décrit plus loin une petite société de buveurs d'eau qui se réunit à l'époque autour de deux sources minérales : "La source de Vaugarni, près le Rosais, et celle de la Fontaine-aux-Eaux, en Dinan. " Bien identifiées, ces fontaines ont existé ou existent encore. La première est située près du Rosais, lieu-dit donnant son nom à une des plages de SaintServan qui se situe au sud de la plage des Fours-à-Chaux; la seconde se trouve près de Dinan dans le creux du vallon de l'Argentel. Cette dernière était très fréquentée par l'élite locale et dans les années 1850, des visiteurs français et anglais provenant des stations balnéaires proches (Saint-Malo, Dinard) excursionnent jusqu'à Dinan afin de goûter cette eau ${ }^{8}$. Chaque année, à la fin de la saison des eaux, de grandes fêtes sont organisées par la municipalité dinannaise ${ }^{9}$. La source de Vaugarni fortifie de la sorte les quelques amateurs d'eaux minérales. Ceux-ci, nous indique le docte avocat, ont même fait installer " un plancher mobile ${ }^{10}$ " pour pouvoir danser lors de bals souvent fréquentés par les édiles locaux.

Microsociété de type thermal aux installations régulières mais temporaires, tel semble être le profil dans les années 1820-1830 de cette première acculturation d'une élite locale avec ce que nous appellerons ici la « vie mondaine de station ". Ce petit groupe humain dont la composition est mal

5. Eugène Herpin, avocat au barreau de Saint-Malo, fut en 1901 le premier président de la Société d'Histoire et d'Archéologie de l'Arrondissement de Saint-Malo. Historien, il consacre l'expression "Côte d'Émeraude " dont il est l'inventeur, dans La Côte d'Émeraude, Saint-Malo et ses souvenirs, Saint-Malo, 1894.

6. Pays de Saint-Malo, en référence au pays d'Alet (Saint-Servan).

7. HERPIN, Eugène, La Côte d'Émeraude..., op. cit. p. 356.

8. L'historien dinannais Yves Castel a fourni de précieux renseignements au sujet de cette fontaine. La première analyse des eaux de cette source date de 1648, par le chimiste Jean Du Hamel, et elles sont dites "parfaite[s] pour soigner les désordres digestifs". En 1764, la fontaine est aménagée, et l'on érige un bassin sous préau. Des curistes célèbres vont y goûter les eaux. Yves Castel cite tour à tour quelques personnalités : Louis Caradeuc de La Chalotais, président à mortier au Parlement de Bretagne, les Anglais William Pitt et Lord Granville. Après la Grande Guerre, la fréquentation devient marginale et un violent orage (16-17 septembre 1929) emporte les installations.

9. Castel, Yves, Chroniques d'Émeraude, Fréhel, Éd. Club-35, 1995, p. 100.

10. HERPIN, Eugène, La Côte d'Émeraude..., op. cit., p. 356-357. 
connue mais dont au moins l'existence est sûre, joue à n'en pas douter un rôle éminent dans la diffusion de ces modes par la multiplicité de ses expériences, tant hydrominérales que balnéaires. Grâce à cette microsociété éclairée, s'enracine aussi en pays malouin le bain de mer. L'élément nouveau, c'est bien cette transition offerte par une pratique plus ancienne, de surcroît originaire, provenant d'" autochtones ", articulant deux vogues ${ }^{11}$ aux tenants et aux aboutissants finalement proches. Dans les deux cas, il s'agit d'une thérapeutique de l'eau qu'elle se décline en crénothérapie ${ }^{12}$, thermalisme ou balnéothérapie.

L'activité balnéaire, au regard de ces faits, ne chasse pas l'activité liée aux sources mais elle complique le tableau et en fait volontiers confondre les acteurs. L'une ne chasse pas l'autre donc, mais l'une peut masquer l'autre, comme semble l'indiquer cet autre texte d'Eugène Herpin :

"Aujourd'hui, cette bonne grand'mère qui se nomme la cité d'Alet, mollement assise sur les bords de la Rance, regarde complaisamment, à travers la verdure de ses boulevards, toutes les juvéniles et élégantes plages de la Côte d'Émeraude, qui ont jailli soudain des ondes fondantes, apéritives, stomachiques ${ }^{13}$ et emménagogues ${ }^{14}$ de sa source chantante de Vaugarni ${ }^{15}$."

Soulignons à nouveau la rareté de ce type de témoignage qui nous offre le chaînon manquant, l'articulation pressentie entre une sociabilité liée aux sources et une acculturation balnéaire. En Bretagne, cette confusion des pratiques semble durer assez longtemps et la différenciation entre ces deux activités prend parfois du temps. Au fil de la recherche, d'anciennes sources sont également apparues. Ainsi l'existence d'une source, dite " Fontaine des Récollets ${ }^{16}$ ", située non loin de la plage de Port-Louis (Morbihan). Nos recherches n'ont pu déterminer si cette fontaine peut être, comme ailleurs, considérée comme un lieu d'acculturation aux eaux, c'est-à-dire si elle a fait l'objet d'une fréquentation importante et régulière par un cercle de buveurs d'eau. Cette fontaine, rapporte un historien de Port-Louis, était située dans l'actuelle avenue des Récollets, qui avait été nommée durant quelques décennies " avenue des Étrangers ${ }^{17}$ " en hommage aux premiers touristes.

11. On ne peut pas à proprement parler de mode, car évoquer la "mode balnéaire " reviendrait à utiliser une expression facile très insuffisante. Or, une mode qui dure plus d'un siècle et demi n'est déjà plus une mode, c'est un mode, un mode de vie.

12. Traitement par les eaux de source.

13. Médicament pour l'estomac.

14. Emménagogue : "médicament ou traitement qui provoque ou régularise la menstruation " (Le Petit Robert).

15. HerPin, Eugène, La Côte d'Émeraude..., op. cit., p. 357.

16. Monmarché, Marcel, Les Guides Bleus - Bretagne, Paris, Hachette, "Les Guides Bleus ", 1930, p. 537. La fontaine tient son nom de la présence d'un ancien couvent, le couvent des Récollets (XVII ${ }^{\mathrm{e}}$ siècle) qui fut transformé en hôpital de la marine, lui-même supprimé en 1926.

17. Buffet, H.-F., Vie et société au Port-Louis, Rennes, Éditions Bahon-Rault, 1972, p. 458. H.-F. Buffet explique que l' " avenue des Étrangers " (plantée d'ormes) a été ainsi baptisée 
Quelques communes du littoral de Loire-Inférieure ${ }^{18}$ possèdent la même originalité que celles évoquées de la région malouine. Elles combinent sources minérales et grèves de sable fin. Le 30 juin 1831, un mémoire sur des travaux réalisés par un maçon entrepreneur de Pornic évoque « un marché de gré à gré passé entre la commune et ledit artisan, pour la réalisation d'un vestiaire et autre établissement sur le bord de la mer, près de l'anse de la Malouine ${ }^{19}$ ". Ce mémoire, apparemment anodin, suscite cependant quelques questions importantes. Ce document sème le doute car à l'examen, ce vestiaire n'est pas, comme il parait l'être, destiné aux bains de mer. Il s'agirait d'un baraquement destiné à abriter des buveurs d'eau minérale. Une autre source de Pornic d'où jaillit une eau ferrugineuse est située tout près de la plage de Gourmalon, nommée aussi (et pour cause) plage de la Source. Dans un premier temps, c'est donc pour son eau (douce) que des touristes fréquentent Pornic : "Les premiers baigneurs furent des buveurs d'eau simplement, et non pas des amateurs de natation ou des partisans de l'eau froide ${ }^{20}$. " Enfin, l'auteur Léon Maitre précise en historien : " L'habitude des bains de mer ne s'est pas répandue tout d'un coup dans les mœurs, elle s'est produite par contact et par entraînement, comme beaucoup de coutumes $^{21}$."

L'exemple pornicais n'est pas isolé dans cette région : les communes de Préfailles, Saint-Michel-Chef-Chef, La Bernerie-en-Retz possèdent elles aussi des sources hydrominérales. À Préfailles, c'est en 1765 que sont pour la première fois précisées par le chimiste Antoine Monnet ${ }^{22}$ les vertus thérapeutiques de la source de Port-Meleu. Les guides relaient les études sur cette source qui " est plus chargée en fer que celle de Pornic, et contient en outre de l'arsenic : on la boit pour l'estomac, l'anémie et le lymphatisme. Elle coule par un robinet, près de la mer qui la recouvre aux grandes marées ${ }^{23}$ ". Vers 1820, " Malmy-en-Pornic attire les buveurs d'eau et la vogue s'étend à la Bernerie, à Saint-Brevin et à [Saint-Michel-Chef-Chef] ${ }^{24}$ ", précisent des historiens locaux.

La transition est ici naturelle entre deux pratiques qui sont véritablement les déclinaisons d'une thérapeutique commune autour des bienfaits de l'eau. Les deux activités vont plus tard se confondre : "En 1860, la belle plage de Saint-Michel-Chef-Chef et sa source d'eau minérale attirent un

en 1886 en l'honneur des baigneurs. Elle reprendra plus tard son nom originel d'avenue des Récollets.

18. Nom, jusqu'en 1957, du département de Loire-Atlantique.

19. Arch. dép. de Loire-Atlantique, 2 O 131-11, Mémoire des travaux exécutés, adressé au Maire par l'entrepreneur (nom illisible) le 30 juin 1831. Cette anse de la Malouine désigne l'anse du Château proche de la pointe de la Malouine.

20. Maitre, Léon, Pornic et la Côte de Retz, de la Bernerie à Saint-Brevin et Mindin, Nantes, Dugas et Cie, 1908, p. 79.

21. Ibidem

22. Article inédit de LÉGAUlT, Maurice, "La source de Préfailles ", octobre 2007, p. 1.

23. Monmarché, Marcel, Les Guides Bleus - Bretagne, op. cit., p. 443.

24. Tessier, Michel, Guillemin, Renée, Saint-Michel-Chef-Chef et Tharon-Plage, La Baule, Éd. des Paludiers, 1987, p. 44. 
grand nombre d'étrangers pendant la saison des bains ${ }^{25}$. " Le baigneur d'alors avait donc tout loisir d'apprécier la revigorante gorgée d'eau minérale, avant de s'immerger dans le " grand bain "...

À Saint-Gildas-de-Rhuys, Saint-Jean-du-Doigt, Saint-Quay-Portrieux ${ }^{26}$ et en bien d'autres points du littoral, des fontaines aux diverses vertus sont répertoriées mais leur rôle " acculturant ", transitionnel, qui seul nous intéresse ici, n'est pas démontré. La carte (Carte 3) proposée relie l'émergence des premières stations balnéaires à la préexistence de sources minérales fréquentées.

\section{Les sources minérales et premières occupations balnéaires dans l'Ouest armoricain avant 1850}

Ces cercles restreints, ces sociétés de buveurs d'eau, vont expérimenter un type de sociabilité caractérisée par « l'entre soi ». Ainsi, à condition que ces sources ne se trouvent pas du jour au lendemain abandonnées, elles sont, en certains points du littoral, l'endroit où se sont développés une acculturation et un goût pour ce qu'on peut appeler commodément la "vie de station ". Bien sûr, cette réflexion ne s'applique qu'à ces quelques lieux bien distincts et il ne faut surtout pas surestimer ce phénomène. Bien des sources traditionnellement fréquentées n'ont pas donné mécaniquement naissance à une station balnéaire. Il demeure néanmoins intéressant de souligner que certains intitulés de syndicats d'initiative réunissent encore au début du XXe siècle ces deux faces d'une même thérapeutique : en 1900, le syndicat d'initiative des communes du littoral lannionais (Côtes-du-Nord ${ }^{27}$ ) prend pour intitulé "Syndicat d'initiative des plages de Perros-Guirec, Trégastel, Trébeurden et des eaux minérales de Lannion ${ }^{28}$ ".

La pratique du bain de mer préexiste à la balnéation maritime. En de nombreux points du littoral, les populations indigènes se baignaient, de façon sporadique, avant que n'apparaissent les premiers aménagements ad hoc. Ce n'est donc pas la baignade qui crée les stations balnéaires mais l'arrivée sur ce littoral de personnes qui veulent prendre des bains de mer, cela à des fins précises, une thérapeutique. Or la balnéation n'est pas inventée et expérimentée par les populations locales, elle est importée sur les plages de la Manche et de l'Atlantique par une élite acculturée. Parmi ces groupes, quantitativement restreints, se trouvent primitivement des aristocrates, à l'origine des premiers aménagements et de la première villégiature balnéaires. Ces cercles aristocratiques, dont le recrutement se réalise par

25. Ibidem.

26. JoAnne, Paul, Itinéraire général de la France - Bretagne 1896, Paris, Hachette et Cie, coll. "Guides-Joanne ", 1896, p. 191. Ces sources sortaient de la falaise et ont donné leur nom à la grève des Fontaines. Leurs eaux, indique le guide, ont été recueillies afin d'alimenter un lavoir.

27. Actuellement Côtes-d'Armor.

28. Gouriou, Anne-Marie, Le tourisme sur la Côte de Granit Rose 1919-1939, Mémoire de maîtrise d'histoire, Université de Haute-Bretagne Rennes 2, 1990, p. 15. 
Carte 3 - Les sources minérales dans l'Ouest de la France

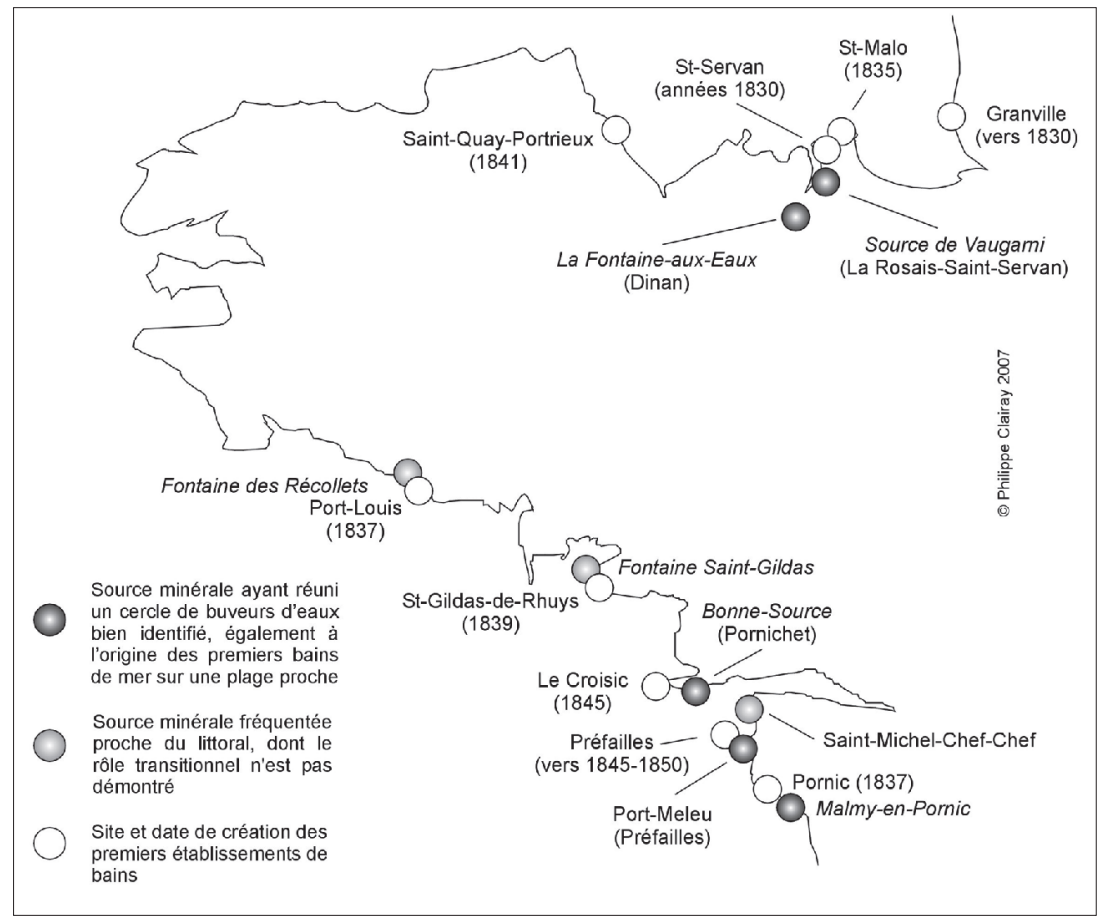

cooptation, vont généralement se fixer sur les points les plus remarquables du littoral, fondant ainsi les premiers noyaux balnéaires. Sont alors privilégiés le lieu d'installation et la possibilité de bénéficier de la meilleure vue sur la mer. Des installations proprement balnéaires sont ensuite aménagées, tel un établissement de bains de mer, indispensable lieu de cure; une digue de protection qui, créée dans un but utilitaire, va devenir un lieu de promenade incontournable; parfois, un établissement de jeu. Les cercles lettrés urbains sont souvent reconstitués au sein de ces stations. Il en est de même du mode de vie qui, malgré des modifications liées à une certaine " décontraction " estivale, est reproduit. À ce moment, l'argument thérapeutique va s'effacer au profit d'un discours plus hédoniste. Ces cercles réunissent ce que Marc Boyer appelle les gate-keepers ${ }^{29}$, les créateurs de distinction. Ces noyaux balnéaires, constitués de manière spontanée, devront ensuite s'organiser afin d'agréger chaque îlot. Les stations balnéaires bretonnes constituées selon ce type seront appelées stations primordiales. C'est le cas de Saint-Malo, où le docteur Chapel crée un établissement de bains, du

29. BoYER, Marc, Histoire du tourisme..., op. cit., p. 19. 
Croisic, de Pornic et de Dinard, hameau développé par les Anglais dans les années 1850.

À cette première phase de création de stations primordiales succède une seconde phase, aux développements géographiques et historiques plus significatifs. Ces stations de la seconde génération sont assez nombreuses en Bretagne. Leur développement va se focaliser sur des plages, parfois à proximité des stations primordiales. De nouveaux groupes sociaux aux desseins plus précis apparaissent et remplacent peu à peu l'ancienne aristocratie balnéaire. Il s'agit de groupes constitués principalement de membres de la bourgeoisie d'affaires d'origine urbaine qui va investir de façon importante, parfois massive, sur le littoral. Se dégagent alors de fortes personnalités qui sauront convaincre d'autres investisseurs, créant ainsi des sociétés chargées du lotissement de ces stations. L'objectif est clairement d'établir aux alentours de belles étendues sableuses des cités (ou des quartiers de ville) à vocation exclusivement balnéaire. Il s'ensuit des modifications dans les choix d'aménagements, avec notamment l'adoption fréquente de plans d'urbanisme.

Ce mouvement s'accompagne d'une spéculation qui elle-même va contribuer à faire connaître certaines localités. De grands projets débouchent sur la création de nouvelles entités balnéaires, soit au sein de stations préexistantes, soit ex nihilo. La plupart des stations balnéaires bretonnes sont nées de ce mouvement qui s'étend des années 1860 au milieu des années 1930. Il s'attache souvent à l'histoire de leur invention une " personnalité-leader ${ }^{30}$ ", véritable créatrice de la station balnéaire. Son œuvre sera déterminante dans l'accession de la cité littorale au statut de station balnéaire reconnue, c'est-à-dire fréquentée par une élite composée de cercles distingués, qui vont à leur tour diffuser la pratique balnéaire. Par imitation, de nouvelles stations balnéaires sont créées jusqu’à la fin des années 1930. À ce moment, la distribution géographique des stations balnéaires semble se figer, cela jusqu'à nos jours, hormis quelques tentatives de fondation dans l'après Seconde Guerre mondiale.

Dinard est la plus ancienne de ces stations balnéaires auxquelles est étroitement associée une personnalité. Issu de l'aristocratie levantine, le comte de Rochaïd Dahdah ${ }^{31}$ dispose de fonds très importants et, à la suite des réalisations britanniques, il va modeler dans les années 1870 de nouveaux quartiers balnéaires à Dinard en concevant un véritable programme urbanistique. À la fois noble, investisseur et spéculateur, il représente, dans notre démonstration, le lien entre les deux étapes susdites. Suivront, sur d'autres plages, de nombreux fondateurs à la personnalité saillante : le journaliste Hébert à Paramé, l'entrepreneur Benoît à Escoublac (La Baule), l'ingénieur Cotard au Val-André, Nabat et Gouin à Saint-Brevin, Marinier et Alix

30. CAZES, Georges, LANQUAR, Robert et RAYNOUARD, Yves, L'aménagement touristique, Paris, PUF, coll. "Que sais-je? ", 1986, p. 52.

31. Un autre personnage a un profil similaire : Sylla Laraque va investir massivement au milieu des années 1880 à Saint-Lunaire. 
à Saint-Cast, Peugeot à Morgat, Brouard à Sables-d'Or-les-Pins, Lajarrige à La Baule-les-Pins. Si entre le premier et le dernier de ces noms s'est écoulée une période de cinquante ans, le processus de développement des stations balnéaires où ils ont œuvré est, fait remarquable, sensiblement le même. Certaines de ces grandes stations sont créées par diverses sociétés dans lesquelles ne se dégagent pas de personnalités-leaders mais dont l'action suit les mêmes principes (Quiberon, Concarneau, Erquy, Bénodet, etc.).

On peut dans certains cas qualifier ces stations de la seconde génération de stations " filles " lorsqu'elles se développent à proximité d'une station déjà constituée, formant avec elle un ensemble de stations balnéaires " en chapelet $^{32}$ ". Saint-Lunaire et Saint-Briac sont de ce point de vue des satellites de Dinard. Parfois, ces stations " filles " vont contester le leadership de leurs anciennes voisines et, à terme, certaines stations primordiales vont perdre leur primauté sur les stations constituées postérieurement à elles. Il en est ainsi du Croisic, supplantée par ses voisines Le Pouliguen, La Baule et Pornichet, et de Saint-Malo par Paramé. Bien évidemment, cette filiation est le plus souvent spatiale et il faut garder à l'esprit que rares sont les cas où l'on peut démontrer une filiation réelle, au sens où les mêmes personnages seraient à l'origine de plusieurs stations. Il n'en demeure pas moins que les sociétés fondatrices de stations balnéaires recrutent leurs membres le plus souvent dans les mêmes couches sociologiques.

La dernière famille de stations balnéaires est celle des stations balnéaires " orphelines ». Il s'agit de plages aux abords desquelles se sont édifiés des villas, souvent le fait d'une bourgeoisie locale, et quelques aménagements touristiques. Elles n'ont généralement pas bénéficié de l'aura de personnalités-leaders et leur histoire balnéaire est difficile à établir. Elles ne sont remarquables, en tant que stations balnéaires, qu'à travers une fréquentation significative et la fidélité des familles qui y ont séjourné. Elles n'ont fait l'objet de grande vague de spéculation et se sont construites de manière discontinue. Malgré leur développement modeste, parfois à l'ombre de leurs grandes voisines, elles constituent le plus grand nombre des stations balnéaires bretonnes. On peut citer, entre autres, Lancieux, Binic, Locquirec, Plougasnou, Saint-Jean-du-Doigt, Brignogan-Plages, Le Conquet, Damgan, etc. Ces stations ne constituent pas à proprement parler une troisième génération puisque leur apparition a pu être concomitante de l'essor des stations de la seconde génération. Ainsi, quelques familles fréquentent déjà Binic ("station orpheline ") lorsque se forme la station balnéaire de Paramé (station de la seconde génération) ${ }^{33}$.

32. Bien avant les travaux des géographes sur ce sujet, le guide Joanne de 1896 parlait déjà de stations satellites : "Les stations balnéaires de la Bretagne, quelquefois isolées, forment le plus souvent des groupes. Sur la côte nord, c'est d'abord et au premier rang Saint-Malo - Dinard - Paramé et leurs satellites, encombrés pendant l'été ", JoAnNE, Paul, Itinéraire général..., op. cit., p. XXIX.

33. La distinction entre ces deux derniers groupes n'est donc principalement fondée que sur une question d'échelle. 
Pour résumer, trois grandes familles de stations balnéaires se dessinent donc : les stations primordiales, souvent fondées par des personnalités-leaders ${ }^{34}$, qui constituent les premières implantations balnéaires aménagées; les stations de la seconde génération se développent dans la foulée des expériences primordiales et forment parfois avec les premières des chapelets de stations (stations "filles"); les stations " orphelines ", sans personnalité initiatrice de l'essor balnéaire, au développement faible et discontinu. Il existe bien sûr une interpénétration entre ces différentes étapes qui impose à l'observateur d'éviter des césures chronologiques trop précises. Certaines stations classées dans la catégorie des stations " orphelines", comme Loctudy ou Le Pouldu, ont bénéficié de l'action d'une ou plusieurs personnalités fondatrices ${ }^{35}$ dont l'action ne permettra cependant pas un essor significatif. Ces stations ne pourront se départir de leur enclavement géographique et/ou de leur manque de notoriété.

Figure 2 - Chronologie des étapes du développement balnéaire en Bretagne

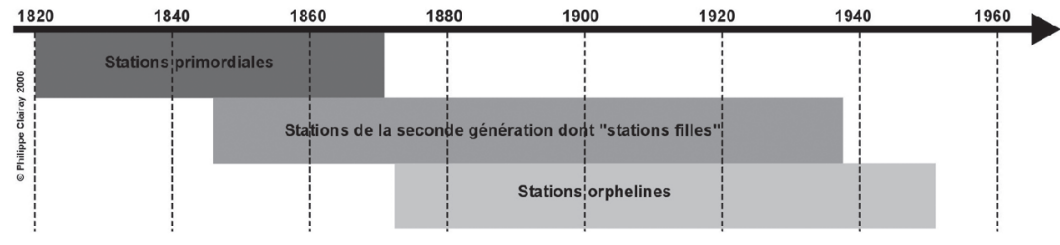

\section{Les évolutions d'une commune littorale à vocation balnéaire}

Les stations balnéaires, souvent dépendantes d'une autre commune, ont subi au cours de leur histoire des destins divers. Quelle que soit la portion de littoral considérée, les stations, au moins à leurs débuts, constituent des noyaux au sein d'une commune. Cette relation symbiotique, originelle, va évoluer au gré du développement de la station. Cette dynamique difficile à appréhender peut cependant être résumée. Ce schéma permet de saisir les trois évolutions possibles d'une station balnéaire, ainsi que celui de la commune dont elle dépend.

Dans le cas 1 (Figure 3), la station balnéaire demeure l'excroissance à vocation touristique d'une commune littorale ou très proche du littoral : elle peut former un quartier important mais spécialisé. Les deux entités (la commune et son quartier balnéaire) vivent de manière symbiotique,

34. Terme utilisé par CAZES, Georges, LANQUAR, Robert, et RAYNOUARD, Yves, L'aménagement touristique, op. cit., p. 52.

35. À Loctudy, on notera l'action de la comtesse de Grandsaignes d'Hauterives qui construit en 1882 les villas de l'anse de Suler à des fins de location aux touristes et au Pouldu celle de l'hôtelier Alphonse Marrec. 
Figure 3-Les trois principales évolutions d'une commune littorale balnéaire à travers l'histoire

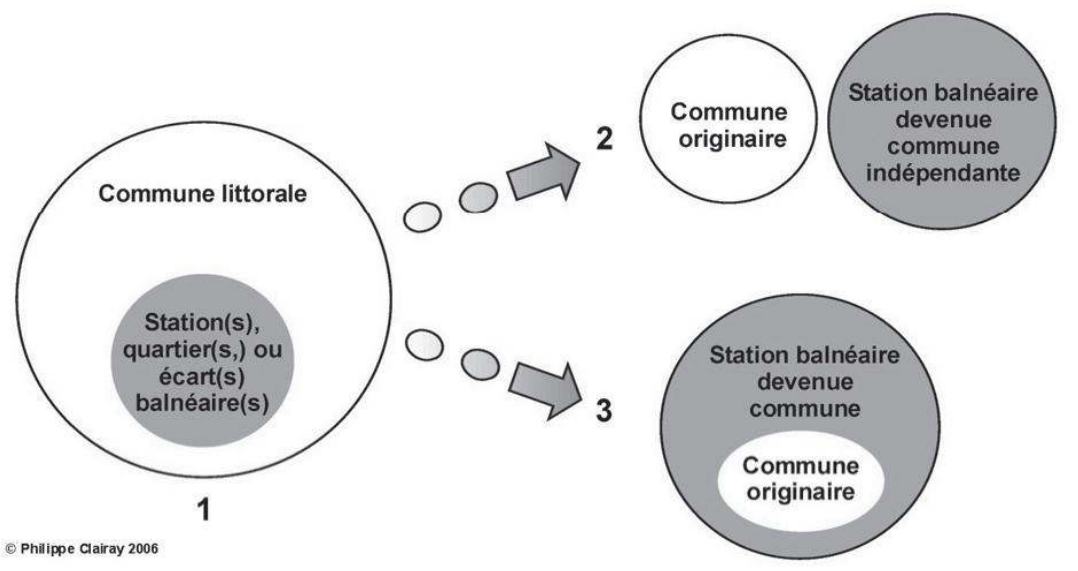

mais dans un même cadre administratif, celui de la commune primordiale. C'est le cas de très nombreuses stations balnéaires bretonnes. Voici les principales cités concernées ${ }^{36}$ : Sables-d'Or-les-Pins (Fréhel), Saint-Lunaire, Saint-Briac, Saint-Cast-Le-Guildo, Pléneuf-Val André, Erquy, Binic, Établessur-Mer, Saint-Quay-Portrieux, Perros-Guirec, Trégastel, Trébeurden, SaintEfflam (Plestin-les-Grèves), Saint-Michel-en-Grève, Locquirec, Carantec, Primel-Trégastel (Plougasnou), Saint-Jean-du-Doigt, Carantec, Roscoff, Le Conquet, Camaret-sur-Mer, Morgat (Crozon), Tréboul (Douarnenez), Loctudy, Beg-Meil (Fouesnant), Concarneau, Le Pouldu (Clohars-Carnoët), Larmor-Plage, Port-Louis, Quiberon, Carnac, Port-Navalo (Arzon), SaintGildas-de-Rhuys, Damgan, Piriac-sur-Mer, La Turballe, Le Croisic, Batzsur-Mer, Pornic.

À partir de ce cas 1, trois évolutions principales sont possibles : soit la station balnéaire demeure jusqu'à aujourd'hui dans cet état, soit elle évolue vers les cas 2 ou 3. Dans le premier cas, la station balnéaire reste attachée à une ville littorale et se développe sans pour autant que son poids supplante celui de la cité originaire. En revanche, si la station se développe significativement, elle peut s'émanciper de la commune originaire et devenir à son tour une commune à part entière. Souvent se trouvent à l'initiative de cette indépendance les personnalités-leaders de la station balnéaire. Deux évolutions sont alors possibles : soit la station balnéaire se juxtapose à la commune originaire (cas 2), soit elle la phagocyte (cas 3). Dans ce dernier cas, la station balnéaire peut donner son nom à l'entité ainsi créée.

36. Sont signalées en italique les stations balnéaires qui dépendent d'une commune, dont le nom est mis entre parenthèses. Dans les cas où la station ne se distingue pas par son nom de la commune originaire, le nom de la commune seule est cité. 
Quelques stations correspondent au cas 2, mais elles sont peu nombreuses. Voici les stations balnéaires devenues villes à part entière, juxtaposées à leur commune originaire ${ }^{37}$ : Le Pouliguen (1854, Bourg-de-Batz), SaintPierre-Quiberon (1856, Quiberon), La Bernerie-en-Retz, (1863, Les Moutiersen-Retz), La Trinité-sur-Mer (1864, Carnac), Bénodet (Perguet, 1878), Pornichet (1900, Saint-Nazaire et Escoublac), Préfailles (1908, La Plainesur-Mer), Larmor-Baden (1924, Baden), Larmor-Plage (1925, Plœmeur), Brignogan-Plages (1934, Plounéour-Trez).

Quelques stations balnéaires bretonnes ont suivi l'évolution décrite dans le cas 3. Dans la plupart des situations, les plages devenues stations balnéaires et ce, à leur fondation, sont des écarts de communes existantes, mais il est rare que la plage " prenne le dessus " sur la cité littorale originaire et lui impose son nom ${ }^{38}$ : Saint-Quay-Portrieux (Saint-Quay-Étables 1860, puis Saint-Quay-Portrieux 1875), Dinard (Dinard-Saint-Énogat 1879, puis Dinard 1921), La Baule-Escoublac (Escoublac puis Escoublac-La Baule 1924, puis La Baule-Escoublac 1962), Pléneuf-Val-André (Pléneuf, puis Pléneuf-Val-André 1965), Saint-Brévin-les-Pins (incluant Saint-Brevin bourg et Saint-Brevin-l'Océan, 1899).

Comme dans toute catégorisation, il existe des cas particuliers. L'agglomération malouine est le plus évident. En effet, au début de l'histoire balnéaire de la Côte d'Émeraude, de proche en proche, chaque cité littorale va développer un quartier balnéaire (cas 1). Les plages de Saint-Malo, SaintServan, Paramé puis Rothéneuf connaîtront un développement balnéaire certain mais dans diverses proportions. Or, toutes ces communes, jadis indépendantes, vont s'associer à Saint-Malo en 1967 (décret du 26 octobre 1967) et former une seule cité très importante (50000 habitants). Cette évolution récente correspond alors au cas 2 . En l'espèce, Saint-Malo-Paramé-Rothéneuf forme un bon exemple de " conurbation balnéaire " bretonne ${ }^{39}$.

\section{Le développement en chapelet des stations balnéaires}

À l'observation, les diverses localisations font apparaître parfois un développement de stations balnéaires en chapelet. Dans un des chapitres de son mémoire de DEA, Philippe Clairay proposait de comparer les évolutions des stations balnéaires bretonnes et anglaises ${ }^{40}$ et avait mis en valeur notamment la variabilité des rapports de force entre des stations

37. La date de la création de la nouvelle commune figure entre parenthèses, suivie du nom de la (ou des) commune(s) originaire(s).

38. La liste donne le nom actuel des communes, et entre parenthèses, la date du (ou des) changement(s) de nom.

39. La seule, car si la frange du littoral s'étendant du Pouliguen à Pornichet apparaît comme un ensemble uni par les diverses élévations, les communes existent encore et n'ont pas (encore?) l'intention de former une seule entité.

40. Clairay, Philippe, Le développement touristique de la Côte d'Émeraude (partie Ille-etVilaine), depuis le second Empire, Mémoire de DEA d'Histoire, sous la dir. de Roger Dupuy, Université de Haute-Bretagne Rennes 2, 1997, p. 15-31. 
qui se développent à proximité les unes des autres. Brighton aura elle aussi ses "filles " avec Eastbourne, Newhaven, Hastings. Dans un second temps se développeront les stations du Yorkshire comme Scarborough, du Lancashire comme Blackpool ou du Dorset comme Poole, Bournemouth, Weymouth, Lyme Regis etc. ${ }^{41}$. Les stations évoquées sont construites selon un modèle programmé, les promenades se font sur un boulevard de planches et sur les piers ou jetées-promenades avec comme fond de scène la mer. Or ce même mode de développement se déploie en Bretagne, notamment sur la Côte d'Émeraude. Dinard va bénéficier à l'ouest de satellites, véritables stations "filles". Il ne faut cependant pas tirer de cette comparaison une mécanique du développement balnéaire : il n'est pas sérieux de dire que cette géographie procède d'une volonté des fondateurs (nous n'en avons aucune preuve!) mais simplement il s'agit de constater et de questionner cette similitude de localisation. Cette singularité n'est pas exclusive des rivages sud de l'Angleterre, ni du littoral breton. Ce développement en chapelet existe également sur le littoral girondin : Arcachon, station primordiale et ses "filles ", Pyla-sur-Mer, Andernosles-Bains, La Teste-de-Buch ou Cap-Ferret. Il en est de même sur le littoral normand avec Granville, suivie de ses satellites Donville-les-Bains, Saint-Pair-sur-Mer, Carolles, entre autres. Plus au nord, sur la Manche, Trouville puis Deauville endosseront tour à tour le rôle de station phare et Houlgate, Villers-sur-Mer, etc. seront fondées dans un second temps. Sur le littoral basque, Biarritz constitue la station primordiale et SaintJean-de-Luz, Hendaye, Anglet, Guéthary, Bidart, des stations satellites. Sur la Méditerranée mais cette fois bien plus tardivement (dans les années 1920), un même développement s'opère : Juan-les-Pins devient la station balnéaire phare ${ }^{42}$, suivie de nombreuses imitations... Il est intéressant de constater que si en Bretagne la géographie des stations balnéaires semble se figer à la fin des années 1930, sur le littoral méditerranéen l'inverse se produit. En effet, de nouvelles créations balnéaires émaillent non seulement les années 1930 mais aussi (et avec quelle vigueur!) les décennies 1950-1960. On peut citer par exemple le fameux port de Saint-Tropez, à l'essor fulgurant, où ce ne sont plus des aristocrates mais des vedettes du cinéma qui sont les inventeurs de distinction ${ }^{43}$. Et l'hypothèse d'analyse, pour l'ensemble des cas énumérés ici, fait appel au processus sociologique de distinction imitation. Les créateurs fondent les stations primordiales, les pasticheurs leurs satellites.

41. Lire à ce sujet le remarquable travail de ALAn-PATMORE, John, Land and leisure in England and Wales, Newton Abbot, South Devon House, David \& Charles Publishers Limited, 1970, 315 p. L'auteur, fort d'une analyse statistique et géographique impressionnante, n'indique cependant pas clairement par quel processus s'est fait ce développement en chapelet.

42. Boyer, Marc, L'invention de la Côte d'Azur, Paris, Éd. de l'Aube, 2002, p. 357-360. Marc Boyer explique le retard pris dans le lancement de la saison estivale sur le littoral méditerranéen.

43. Certes l'" élite " change mais le processus demeure identique. 
À travers deux exemples régionaux apparaît de manière plus manifeste cette caractéristique du développement en chapelet de certaines stations balnéaires. En Bretagne, deux régions connaissent cette implantation particulière, la Côte d'Émeraude et la presqu'île de Guérande. Le schéma suivant se propose de résumer ce développement, observé dans sa globalité à la fin de notre période de recherche en ces deux points du littoral breton.

Figure 4 - Le développement des stations balnéaires en chapelet : les exemples de la Côte d'Émeraude et de la Côte d'Amour

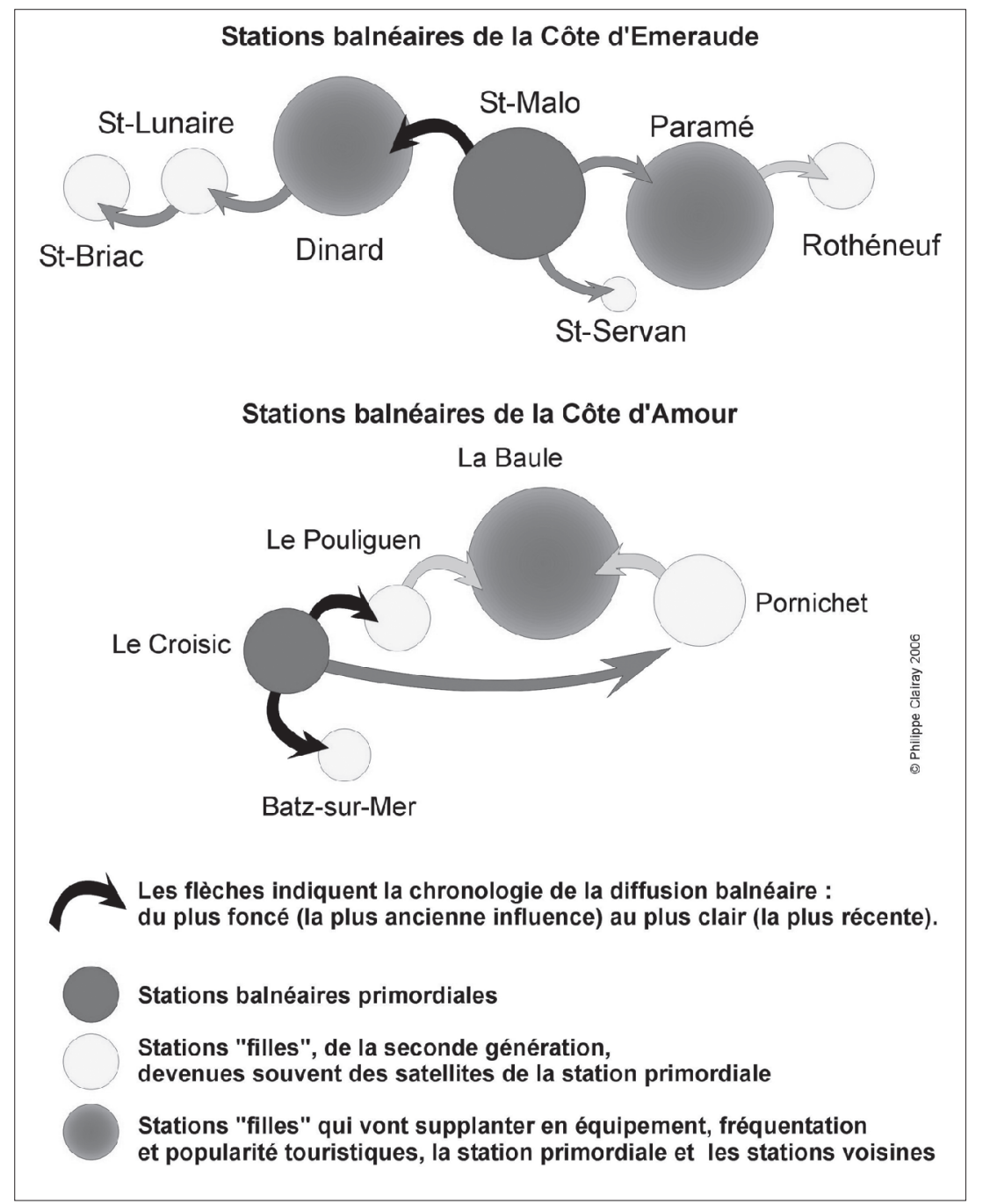


Constatons que ces deux grandes régions balnéaires sont également les plus anciennes de Bretagne. De plus, elles forment encore aujourd'hui les deux plus importantes zones de tourisme balnéaire de la région bretonne. L'image la plus appropriée, telle qu'elle peut apparaître après analyse, est celle d'un développement par racines adventices. À partir de ces points nodaux que constituent les stations primordiales, des plages plus ou moins proches (en fonction bien sûr de la géomorphologie des côtes) sont colonisées par imitation. Mais ces stations de la seconde génération se développent généralement de manière bien plus importante et plus rapide. En effet, les créateurs ne sont plus les mêmes. Les aristocrates de la première génération de station cèdent le pas à des personnages marquants, grands financiers de la bourgeoisie locale ou nationale, artistes renommés ou puissants rentiers, qui vont lancer ces plages. Ce faisant, ils déplacent le centre mondain des stations primordiales et donc transfèrent le pouvoir d'attraction (c'est-à-dire l'aura, la renommée, la distinction) des premières créations vers les secondes. Par un effet de feed$b a c k$, le développement devient sinon exponentiel, du moins très intense.

À ce stade, deux évolutions sont possibles. Dans le premier cas, si les stations de la seconde génération ne subjuguent pas les premières nées, elles leur font largement concurrence, d'autant plus qu'elles en sont proches (c'est le cas du Pouliguen par rapport au Croisic dans les années 1860 et plus tard de La Baule par rapport au Pouliguen et Pornichet). Dans le second cas, elles dépassent les stations primordiales en équipements, fréquentation et renommée, et deviennent à leur tour des stations phares qui peuvent faire naître des satellites (Paramé influence largement le développement de Rothéneuf). Ce qui est valable à ce niveau local l'est également au niveau régional. Sans cesse, au cours de cette histoire balnéaire, sera discutée la primauté des grandes stations.

Le processus de développement est constitué de plusieurs mouvements distincts dans le temps. Dans le cas des stations balnéaires de la Côte d'Émeraude, il est évident que la ville de Saint-Malo, station primordiale, a contribué au développement de Paramé, sa proche voisine, mais ce n'est pas seulement en lui fournissant ses baigneurs. La cité malouine, en fixant la première des groupes de baigneurs, a suscité la création de la station paraméenne grâce à un processus d'acculturation de ces mêmes villégiateurs. Et la grande plage de Paramé, par imitation de la faible mais aristocratique station malouine, sera développée par un consortium de journalistes composé de membres éminents de la bourgeoisie parisienne.

Avant Paramé, Dinard, de l'autre côté de la Rance, a connu un important essor balnéaire. Or, parmi les fondateurs britanniques de Dinard, se trouvent des consuls anglais en poste à Saint-Malo qui vont, en franchissant l'estuaire, coloniser le rivage dinardais et y accueillir leurs concitoyens. Saint-Malo est donc indirectement à l'origine de ce développement. De plus, outre le décisif apport britannique, Dinard va bénéficier de l'arrivée du personnage phare du Saint-Malo balnéaire, M. Legros, célèbre guide baigneur qui, après s'être brouillé avec la municipalité malouine, franchit également la Rance et se fixe à Dinard, contribuant par sa popularité au succès de la jeune station. Ainsi, 
tout autant que les contextes culturels, ce sont de petits événements qui ancrent les pratiques et impulsent ou amplifient le développement.

Il en est de même pour les stations de la presqu'île de Guérande. L'exemple du Croisic va conduire une élite cette fois plutôt régionale à créer de nouvelles stations. Ce sera tout d'abord le Pouliguen, puis Pornichet. Si les initiateurs du Pouliguen se composent de membres de la bourgeoisie en majorité régionale, à Pornichet, les investisseurs les plus puissants sont des artistes lyriques et des libraires-éditeurs. Comme dans le cas malouin, Le Croisic ancre la pratique et les stations de la seconde génération la développent. Plus tard, ce sera la grande dune que représente le littoral baulois qui sera conquise et aménagée. À chaque fois, les îlots balnéaires primordiaux s'installent sur les points les plus remarquables du littoral (généralement un éperon rocheux ou un site particulièrement spectaculaire, par rapport au reste de la côte), ce qui conduit Françoise Hamon à écrire, avec juste raison : « Le caractère primordial n'est pas la commodité du site, mais la vue ${ }^{44}$. "Ce n'est que dans un second temps que seront mises en écrin les grandes plages comme La Baule. Suivant cette réflexion, il pourrait surgir une contradiction entre ces localisations et la pratique du bain de mer : pourquoi ne pas s'installer tout près des flots, au " rez-de-marée "? Encore pour des raisons culturelles. La plage, aux premiers temps de cette villégiature, n'a d'intérêt esthétique qu'en tant que décor. Plus que son voisinage immédiat, c'est sa vue que recherchent ces villégiateurs, autant qu'ils désirent être vus. À Dinard, ce sont les quartiers du Moulinet et de la Malouine (situés sur des pointes) qui se développent le plus tôt, quand les abords de la plage de l'Écluse demeurent inoccupés; sur le littoral du sud de la Bretagne, Le Croisic, site rocheux, se développe avant La Baule, plage plane. Les modifications de cette topographie balnéaire seront également les conséquences d'un changement sociologique. En effet, c'est lorsque les bourgeoisies du Second Empire puis de la III ${ }^{\mathrm{e}}$ République gagneront le littoral que s'opérera le basculement. Les plages, jusqu'alors considérées comme des moyens, changent de statut symbolique et deviennent des fins en soi : elles seront donc investies (dans tous les sens du terme) et mises en écrin par de nombreuses constructions et divers équipements annexes. Et là encore, suivant le paradigme d'imitation/distinction, chacun tentera de rivaliser avec l'autre, mais cette fois, sur la plate ligne du rivage. C'est ainsi que le premier rideau de villas, le front de mer, sera pour longtemps le plus estimé, tandis que les deuxièmes et troisièmes rangs n'auront qu'un intérêt relatif.

\section{Une typologie de l'organisation contemporaine des stations balnéaires}

S'inspirant d'observations faites sur le terrain et adaptant pour les cas bretons les typologies proposées par des géographes comme Georges

\footnotetext{
44. Hamon, Françoise, "L'architecture balnéaire sur la Côte d'Émeraude ", Annales de la Société d'Histoire et d'Archéologie de l'Arrondissement de Saint-Malo, Saint-Malo, 1981, t. LVIII, p. 7.
} 
Cazes, ce schéma regroupe en trois cas les types d'organisation spatiale des stations balnéaires. Bien entendu, il s'agit d'une image figée qui ne doit pas être lue comme immuable.

Dans le cas 1 (Figure 5), la cité littorale ne possède qu'un quartier ou écart balnéaire et sera dite unipolaire. C'est, en Bretagne, un type de station très répandu, avec les exemples de Morgat (Crozon), Tréboul (Douarnenez), PléneufVal-André, Le Pouldu (Clohars-Carnoët), Les Rosaires (Plérin), Camaret-sur-Mer, Damgan, Le Pouliguen, La Bernerie-enRetz, Quiberon, Port-Louis, Saint-PierreQuiberon, Larmor-Plage, Préfailles, La Trinité-sur-Mer, Saint-Gildas-deRhuys, Saint-Michel-en-Grève, SaintEfflam (Plestin-les-Grèves), Carnac, Binic, Roscoff, Bénodet, Port-Navalo (Arzon), Piriac-sur-Mer, La Turballe, Saint-Marc-

Figure 5 - Une typologie de l'aménagement des stations balnéaires contemporaines - Cas 1

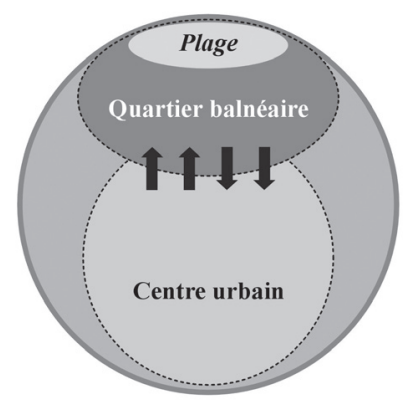
sur-Mer (Saint-Nazaire).

Dans le cas 2 (Figure 6), la ville littorale possède plusieurs quartiers ou écarts balnéaires qui contribuent largement à son essor économique. Ces stations seront dites multipolaires. C'est le cas en Bretagne de Saint-Malo, Saint-Lunaire, Saint-Briac-sur-Mer, Saint-Cast-le-Guildo, Saint-Jacut-de-la-Mer, Erquy, Étables-sur-Mer, Perros-Guirec, Trégastel, Trébeurden, Carantec, Concarneau, Locquirec, Pornic, Brignogan-Plages, Saint-Michel-Chef-Chef, Saint-Quay-Portrieux, Pornichet, Loctudy, Plougasnou, Fouesnant, Le Conquet. À terme, ces cités balnéaires, qu'elles soient unipolaires ou multipolaires, pourront connaître un développement de leur activité touristi-

Figure 6 - Une typologie de l'aménagement des stations balnéaires contemporaines - Cas 2

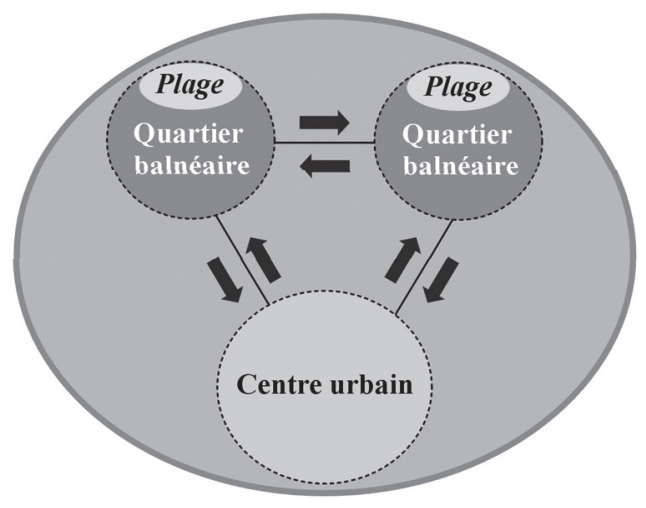


que comparable aux stades 1, 2 ou 3 de la figure 3 . Le poids du tourisme balnéaire dans leur activité économique peut être variable en fonction des équipements et de la fréquentation touristiques mais il n'est jamais négligeable. En majorité les stations balnéaires bretonnes s'apparentent à ce modèle.

Dans le cas 3 enfin, la station balnéaire a été fondée ex nihilo, selon un plan d'urbanisme. Elle est très dépendante du tourisme qui constitue sa principale activité et a généralement supplanté sa commune de tutelle, qui dépend à son tour entièrement du destin économique de la station balnéaire. Ne sont concernées par ce modèle que quelques rares stations bretonnes telles que La Baule-les-Pins, Saint-Brévin-les-Pins et Sables-d'Orles-Pins ${ }^{45}$.

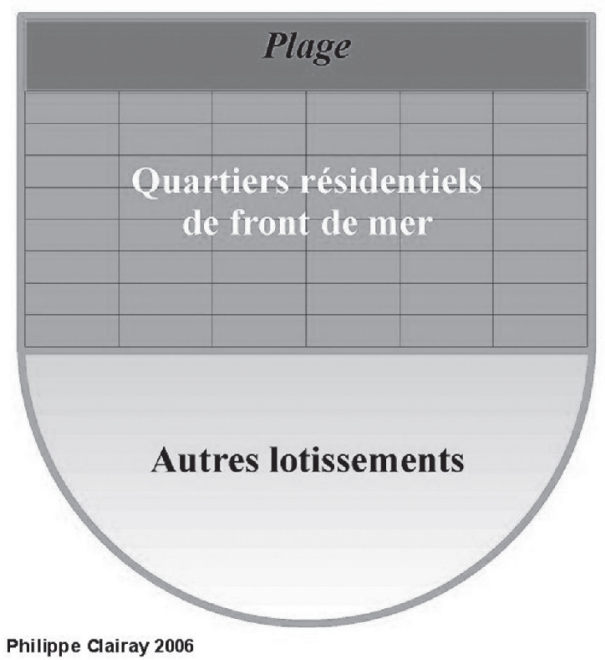

Figure 7 - Une typologie de l'aménagement des stations balnéaires contemporaines - Cas 3

\section{Le tourisme balnéaire, créateur d'images de la Bretagne}

L'influence touristique a été double, dans la forme autant que dans le fond. La typologie des stations balnéaires bretonnes démontre que les projets d'aménagement du littoral ont été multiples et divers. Pourtant, au-delà de ces particularismes, le tourisme a su développer un discours commun en imprégnant l'imaginaire breton.

45. La station balnéaire de Sables-d'Or-les-Pins est citée dans cet exemple car, bien que rattachée aujourd'hui à la commune de Fréhel, elle est la principale activité et principale source de revenus de la commune. 


\section{Un littoral échappant aux promoteurs}

La Bretagne a l'image d'un littoral qui a échappé aux promoteurs immobiliers. Pourtant, les projets et les réalisations n'ont pas manqué. En Bretagne, les grands lotissements balnéaires se mettent en place en deux temps, des années 1880 jusqu'à la Première Guerre mondiale et dans les années 1920. Les premières créations complètes des stations balnéaires bretonnes font partie d'un mouvement français plus général. Le nombre de sociétés immobilières explose au niveau national, après les lois de 1863 et de 1867 qui suppriment le contrôle des pouvoirs publics lors des créations ${ }^{46}$. Les côtes bretonnes n'ont pas échappé aux promoteurs et les réalisations du $\mathrm{XIX}^{\mathrm{e}}$ siècle sont indéniablement des réussites pour leurs commanditaires. $\mathrm{Au} \mathrm{xx}$ e siècle, les entreprises immobilières souffriront de la Première Guerre mondiale et de la crise de 1929. Les réalisations interviennent néanmoins plus tardivement que les lotissements des plages normandes. Les préjugés demeurent défavorables à la Bretagne. En 1858, Alfred Dumesnil sollicite Jules Michelet en villégiature à Pornic pour qu'il lui trouve une domestique bretonne, une «femme ou fille d'un pays relativement plus pauvre que la Normandie qui trouvât avantage dans ce déplacement ". Jules Michelet l'informe néanmoins que Pornic est un pays intermédiaire où la main-d'œuvre est mieux payée ${ }^{47}$. Les premiers lotisseurs ne recherchent pas frénétiquement en Bretagne des plages à exploiter. Le comte Hennecart ne crée une station balnéaire à La Baule que parce qu'il découvre un site vierge superbe et facilement exploitable : il est le principal actionnaire de la ligne de chemin de fer Saint-Nazaire - Le Croisic ouverte en 1879 et peut donc aisément acheter les parcelles où il installe à proximité la gare ${ }^{48}$.

De plus, rares sont les projets bretons de création ex nihilo qui ont un simple but spéculatif. La station balnéaire de Morgat, réalisée par la famille Peugeot à partir de 1884, est ainsi plus un jardin pour les fondateurs qu'une station balnéaire, au point que Michèle Kerspern et Daniel Le Couëdic la qualifient de "Vésinet littoral ${ }^{49}$ ". Cette démarche s'appuie généralement sur un architecte, choisi pour homogénéiser la station. Le comte Hennecart demande à l'architecte Georges Lafont de lui dessiner le plan de La Baule, Armand Peugeot confie aux architectes Abel et Gaston Chabal la réalisation de nombreuses villas. Par rapport à la longueur du littoral, les projets de ce type en Bretagne sont minoritaires : Paramé, Sables-d'Or-les-Pins, SaintEnogat, Morgat, La Baule, peut-être Caroual-Plage et Carnac-Plage. À une

46. FougniÉ, Sébastien, La Mutation contemporaine des stations balnéaires françaises : tentative de modélisation de la station littorale intégrée, Thèse de Doctorat de Géographie, Université de Nantes, 2006, p. 223.

47. MichELET, Jules, Correspondance générale, t. VIII 1856-1858, 1998, Lettre d'Alfred Dumesnil, du 4 septembre 1858, p. 753 et Lettre à Alfred Dumesnil, du 6 septembre 1858, p. 755 .

48. NENNIG, Jean-Pierre, Le Chemin de fer Nantes-Le Croisic et Guérande (1851-2001), Nantes, Ouest éd., 2001, 175 p.

49. Kerspern, Michèle, et LE COUËDIC, Daniel, " Le mode balnéaire : Gaston Chabal à Morgat ", Modernité et régionalisme en Bretagne (1918-1945), Liège/Bruxelles, Pierre Mardaga éd., 1986, p. 92. 
époque où le regard du lotisseur peut être jugé intrusif (la loi sur le permis de construire n'est étendue aux villes de plus de 2000 habitants qu'en 1902, à toutes les communes en 1943), de telles mesures sont nécessairement ressenties par la population. Or peu parviennent à " lancer une plage ". Même ces stations sortent du cadre strict du lotissement. Le projet du comte Hennecart (une station balnéaire familiale comme celle de Blankenberg près d'Ostende) évolue après son décès, en 1884 : son associé Édouard Charlu adjoint au premier lotissement à trame orthogonale un quartier des Oiseaux à la mode anglo-saxonne centré sur un jardin public ${ }^{50}$. La station balnéaire de La Baule sera finalement structurée par quatre grands lotissements (Benoît, Pavie, Hennecart-Darlu, La Baule-les-Pins). Face à aussi peu de grands projets, le littoral apparaît pour le touriste dans sa grande majorité comme un espace de liberté : la station balnéaire bretonne n'est pas représentée comme un vaste lotissement.

Enfin, les promoteurs ne sont pas les premiers à investir dans les stations balnéaires bretonnes. Le marchand de biens parisien André Bernheim, connu pour être un lotisseur averti à Rennes (rue de Fougères), Tréboul, Penfoul et Concarneau (aux Sables-Blancs), arrive tardivement en Bretagne, comme sur la Côte d'Émeraude où il se contente de relancer l'activité du Grand Hôtel de Paramé ${ }^{51}$. Sa société s'insère en fait dans un processus de vente bien établie. À Tréboul, elle achète plusieurs hectares de parcelles entre 1926 et 1930 à des autochtones, dont la famille de conserveurs Le Guillou de Penanros. Son achat porte cependant sur des parcelles proches de la mer, que certains ont déjà commencé à diviser pour lotir : en 1926, la veuve de Théophile Guillou de Penanros divise une de ses parcelles en dix-neuf lots. Néanmoins, la superficie de ces terrains permet de nouveaux partages, certains sont revendus en un peu moins de vingt lots ${ }^{52}$. Par contre, à Concarneau, sa société immobilière n'achète que quelques petites parcelles, par exemple en 1937 à Marcel Péan, chef d'atelier angevin, qui avait déjà procédé à une multitude de partages entre 1928 et $1931^{53}$. La Bretagne connaît en fait une multitude de petits lotissements, de la part tant des autochtones que des personnes étrangères à la commune, qui ne crée pas d'unité de la station balnéaire mais au contraire facilite le dispersement de l'habitat.

\section{Une dimension maritime touristique}

Faute d'action forte des promoteurs, les images touristiques, qui sont le produit d'une époque, vont naturellement associer la Bretagne au maritime.

50. Charles, Alain, " Dans le doux dédale des dunes ", 303. Arts, recherches et créations, $1^{\text {er }}$ trimestre 1994, p. 32.

51. Delignon, Gaëlle, Saint-Malo-Paramé; Urbanisme et architecture balnéaire (1840-1940), Rennes, PUR, 1999, p. 130.

52. Arch. dép. du Finistère, 3 P 285/8, Tréboul, matrices cadastrales non bâties (19141947).

53. Arch. dép. du Finistère, 3 P 42/13, Concarneau, matrices cadastrales non bâties. 
Au début du XIX ${ }^{\mathrm{e}}$ siècle, les touristes s'installent dans les villages littoraux qui peuvent les accueillir, ports où le voyageur trouve la possibilité de se loger et de se nourrir sur une longue période. Les équipages des bateaux de passage ont déjà fait naître un marché locatif, bien avant la venue sur les côtes de personnes désireuses de prendre des bains de mer. Les premières stations balnéaires de Bretagne ont quasiment toutes ce profil : Saint-Malo, Le Croisic, Pornic, Port-Louis sont des ports, bien souvent de cabotage. Les premiers voyageurs s'y dirigeant par commodité, la dimension maritime de la Bretagne va fortement s'ancrer dans la mentalité touristique. Elle est renforcée par l'afflux sur les côtes depuis le XVIII ${ }^{\mathrm{e}}$ siècle des populations miséreuses de la Bretagne intérieure surpeuplée et la course à l'équipement portuaire de la part des petits ports au XIX ${ }^{\mathrm{e}}$ siècle $^{54}$. Quand les touristes arrivent sur les côtes bretonnes, le littoral apparaît comme un espace dynamique, où les Bretons trouvent à vivre. De plus, le logement chez l'habitant oblige les baigneurs à côtoyer les autochtones. Hippolyte Etiennez observe en 1858 qu'on trouve au Pouliguen " peu d'hôtelleries, il faut se loger chez les habitants ${ }^{55}$ ".

En plus d'une ville, le port possède d'autres atouts : c'est un lieu de flânerie et de spectacle. Alain Corbin, analysant le rapport du voyageur au port, constate qu'au temps de l'Encyclopédie, le port se présente comme un lieu instructif, propice aux leçons de choses de la mer, mais qu'à partir de la Restauration, la rituelle promenade des quais se transforme. En 1836, Victor Hugo « déteste toutes ces maçonneries dont on caparaçonne la mer. [...] Plus le port est petit, plus la mer est grande ${ }^{56}$ ". Les quais deviennent des promenades. Au Croisic, Jean-Charles Caillo invite à se lever entre cinq et six heures du matin : "C'est l'heure du départ des bateaux de pêche. Toute une petite flotte aux voiles diaprées de blanc, de rouge, de brun, se déploie dans le chenal; allez jouir de ce coup d'œil sur les montagnes de Lénigo et de l'Esprit, ou rendez-vous sur la chaussée du Tréhic qui s'étend en mer de près d'un kilomètre. [... Puis] vers les deux à trois heures, recommencez à parcourir les quais animés par le retour des bateaux, le mouvement commercial du port, l'entrée et la sortie des navires ${ }^{57}$. "

Les conseils municipaux sont d'ailleurs attentifs à ce que les quais restent une promenade agréable. En 1897, celui du Croisic décide de les arroser durant les mois de juillet et d'août car « les quais du Croisic deviennent

54. MASson, Philippe, Grandeur et misère des gens de mer, Lavauzelle, coll. « L'Histoire, le moment ", 1986, p. 76 et GESLIN, Claude, "Les transformations des ports secondaires bretons au XIXe siècle ", La Vie industrielle en Bretagne. Une mémoire à conserver, Rennes, PUR, 2001, p. 147-162.

55. ETIENNEZ, Hippolyte, Guide du voyageur à Nantes et aux environs, Nantes, Éd. Petitpas, 1858, p. 257.

56. Hugo, Victor, Euvres complètes, t. V, Paris, Gallimard, Paris, p. 1108, cité par CoRBIN, Alain, Le Territoire du vide; L'Occident et le désir du rivage (1750-1840), Paris, Aubier, 1988, p. 223.

57. CAlLLO, Jean-Charles, Le Bateau à vapeur et les bains de mer; Voyage de Nantes au Croisic en 1845, Nantes, Éd. joca seria [reéd. 1993], p. 56-57. 
pendant la saison des bains le lieu de passage d'un très grand nombre de voitures et une affluence de promeneurs lesquels, principalement pendant les jours de forte brise, sont énormément incommodés par la poussière que soulève le vent ${ }^{58}$ ". Néanmoins, l'enchantement du touriste se concentre sur l'animation et les couleurs, le port est transformé en décor. Les touristes détournent l'usage des infrastructures portuaires. À Saint-Malo, on affectionne le Môle : "Quand la mer est haute, on s'y croirait dans un vrai navire et nous avons vu des personnes qui se payaient un commencement de mal de mer en regardant le balancis des mâts des bateaux affourchés sur leurs tangons ${ }^{59}$. "Afin de proposer ce type d'animation, des stations balnéaires créées ex nihilo et donc dépourvues de port vont s'équiper. La Société anonyme de La Baule construit en 1892 une estacade de bois de 85 mètres de longueur, dépeinte par Émile Auzou comme une grosse estacade enlaidissant la plage, s'étendant trop peu en mer pour qu'on aille y rêver et sur terre pour qu'on y monte à toute heure de marée ${ }^{60}$. La station balnéaire investit dans l'imaginaire maritime ${ }^{61}$.

Les crises d'adaptation que connaissent les petits ports de cabotage et de pêche permettent à la plaisance de s'implanter à partir de la seconde moitié du XIX ${ }^{\mathrm{e}}$ siècle dans les nombreuses installations en Bretagne, véritable " poussière portuaire " favorisée alors par l'Administration. Tandis que l'activité balnéaire devient l'activité principale de la commune, le conseil municipal du Pouliguen ne parvient pas à moderniser son port, désormais incompatible avec le tonnage des bateaux de commerce. Les régates qui débutent au Pouliguen en 1875 ont pour but de fédérer le port et la station balnéaire. La plaisance s'installe progressivement dans le port. Au mois de février 1883, le conseil municipal demande " aux nombreux bateaux de plaisance et aux bateaux de pêche de s'amarrer sans gêner les mouvements des navires à l'entrée et à la sortie du port ${ }^{62}$ ". Les petits ports vont être recyclés en port de plaisance, ce mouvement s'accélérant avec la popularisation de la plaisance après les années 1950. La multiplication puis l'abandon par les professionnels de la mer, dès la fin du XIX ${ }^{\mathrm{e}}$ siècle, des petites entités portuaires a conforté le dispersement touristique, en offrant de nouveaux cadres où s'installer.

\section{Un tourisme modeste}

Face à la multiplication des stations balnéaires, la plupart d'entre elles se considèrent comme des plages familiales. Se qualifier ainsi est intéres-

58. Délibération municipale du Croisic, du 7 mai 1897.

59. BouRDAs, Albert, Guide du golfe de Saint-Malo, ou 15 jours à la côte d'Émeraude, 1894, p. 30 .

60. Auzou, Émile, La Presqu'île de Guérande; Étude géographique, historique et économique; Manuel des touristes et des baigneurs, Paris, Plon, 1897, p. 184.

61. VINCENT, Johan, L'intrusion balnéaire; Les populations littorales bretonnes et vendéennes face au tourisme (1800-1945), Rennes, PUR, coll. " Histoire ", 2007, p. 131-135.

62. Délibération municipale du Pouliguen, du 25 février 1883. 
sant pour plusieurs raisons. Tout d'abord, la plage familiale est un signe de sécurité. En 1887, Léon Trebuchet, détaillant son voyage à Belle-l̂le-en-Mer, indique qu'il «n'existe pas le moindre galet sur ces plages, mais le sable le plus fin, le plus solide et les plus doux aux pieds. La mer se retire très peu. Aucun danger n'est donc à redouter; aussi voit-on nombre d'enfants s'ébattre sous les yeux de leurs mères, du matin au soir, sur l'étendue de ces plages ${ }^{63}$ ". La sécurité du bain est un aspect essentiel dans une station balnéaire. Bien qu'il existe des guides-baigneurs, des cordes de sécurité et des barques de surveillance, un site sûr est considéré comme la meilleure solution contre la noyade. La plage du Nau, au Pouliguen, possède une pente douce, sans pierre et sans trou qui puisse faire trébucher : " aux nageurs, à ceux qui aiment à lutter contre la vague, d'aller plus loin sur la grande côte; c'est ici la plage des enfants ${ }^{64}$ ". Le sable de la plage de PortMaria à Quiberon « lui donne une sécurité absolue; [...] c'est la plage rêvée pour les enfants ${ }^{65}$ ".

La plage familiale représente également un rempart contre le snobisme. Henri Corsin énumère les joies saines que l'on trouve au Croisic, parmi lesquels, "pour les mamans et les enfants, d'innombrables petits coins, des plagettes délicieuses, des nids d'ombre ou de soleil, entre les rochers, tout près des flaques sans danger, où toujours quelque chose remue ". Il invite ceux qui veulent profiter d'un casino ou d'un dancing à aller dans les stations balnéaires voisines en vogue ${ }^{66}$. Alors que les stations balnéaires françaises se transforment pour devenir des villes d'agrément et de divertissement avec la découverte à la fin du XIX ${ }^{\mathrm{e}}$ siècle des microbes obligeant à éloigner les malades, les stations balnéaires bretonnes conservent longtemps l'optique médicale des premiers bains de mer. La Bretagne avait d'ailleurs été en pointe, avec l'invention de la cure marine par le docteur Lhoste en 1850 à Saint-Malo, reprise en 1857 par le docteur Perrochaud à Berck-Plage. L'institut marin de Roscoff, inauguré en 1899 par Louis Bagot, est ainsi le plus vieil établissement de thalassothérapie moderne de France. C'est dans cet institut que Louison Bobet, en convalescence après un accident de voiture, aura l'idée de médiatiser la thalassothérapie, ouvrant un centre à Quiberon en $1963^{67}$. Cette approche médicale n'intéresse pas le mondain et un établissement médical tel l'Institut Verneuil à La Baule sera en 1906, quelques années après son ouverture, transformé en casino.

63. TRÉBuchet, Léon, Les Étapes d'un touriste en France, Belle-Île-en-Mer, Paris, Hennuyer, 1887, p. 122.

64. Album de la côte guérandaise et ses environs; Quelques renseignements pour les excursionnistes de la presqu'île guérandaise, 1907, p. 53.

65. La Presqu'île de Quiberon: Auray, Carnac, Vannes (Morbihan); Guide pratique du touriste, sans date (années 1920), p. 17.

66. Consin, Henri, Le Croisic. Son port, sa plage, ses côtes, Éd. Syndicat d'initiatives, 1930, p. 49.

67. GALLAND, Nicolas, "De l'héliothérapie à la thalassothérapie ", La Santé et la mer en Bretagne, Rennes, Institut culturel de Bretagne, 1997, p. 104-117. 
Enfin, la plage familiale est un signe de fidélité à la station. Le parisien M. Rossignol se renseigne auprès du maire de Concarneau en 1864. Son questionnement est révélateur de l'état d'esprit d'un touriste père de famille de cinq enfants, qui s'inquiète du choix de l'endroit où il pourra prendre les bains de mer. Outre la qualité de la plage et la facilité de se loger, il demande si le pays est sain, la vie peu chère et si des baigneurs fréquentent déjà chaque année la localité ${ }^{68}$. Faute de guides aisément disponibles à l'échelon national (le premier guide sur la Bretagne de la collection Joanne est publié en 1867), le baigneur se fie au on-dit. Savoir si la localité est fréquentée est un bon indice de la qualité de la station balnéaire. Le Pouliguen, selon son syndicat d'initiative, " est une plage de famille où les jours coulent rapidement et agréablement. Une aimable et fidèle clientèle, qui va s'accroissant sans cesse, lui revient d'année en année, reconnaissant ainsi les efforts considérables qui sont faits pour lui procurer toujours plus de confort et plus d'agrément ${ }^{69}$ ". L'enfant est un facteur de fidélisation de la clientèle. Pour attirer le baigneur, notamment les familles, le coût du séjour est également étudié.

\section{Les " petits trous pas chers »}

Censées être éloignées des grandes villes, les stations balnéaires bretonnes prétendent proposer des séjours bon marché. L'éditeur La Fare crée d'ailleurs à la fin du XIXe siècle une collection afin de promouvoir " les petits trous pas chers", bien qu'il visite toutes les stations littorales, y compris en 1903 Trouville et ses villas à 10000 francs la saison, l'aristocratique Deauville, la mondaine Dinard, rivale de Trouville et de Deauville. Il relaie ainsi le fort engouement pour La Baule qui, selon lui, connait le plus fort développement de toutes les stations balnéaires de la Basse-Bretagne depuis une dizaine d'années. Les prix à La Baule, comparables à ceux qui se pratiquent à Paramé ou à Beg-Meil (entre 400 et 2000 francs pour le mois d'août), restent inférieurs aux plus mondaines des stations normandes. Ils augmentent à partir des années 1910, quand La Baule rejoint le statut, exceptionnel en Bretagne, de Dinard. Les stations balnéaires les plus modestes ont droit à quelques lignes. Malgré la distance, Le Guilvinec est conseillé aux petites bourses car le loyer atteint les 100 à 120 francs pour trois mois. À Saint-Pierre-Quiberon, on peut louer chez l'habitant des chambres pour 40 à 50 francs par mois et des chalets dont le prix varie entre 150 et 500 francs pour la saison. En 1903, les prix des locations dans les stations balnéaires bretonnes sont généralement inférieurs à 1000 francs par mois ${ }^{70}$.

68. Arch. mun. de Concarneau, 4 R 1, Lettre de E. Rossignol (Paris-Batignolles) au maire de Concarneau, du 29 juin 1864.

69. Le Pouliguen. Sa plage, son bois, son port, sa côte, Éd. Syndicat d'initiatives, 1934, non paginé.

70. "Les petits trous pas chers »; Guide des familles aux bains de mer, plages de la Manche et de l'Océan, Paris, La Fare éd., 1903. 
La vie en Bretagne est réputée bon marché. En 1900, Paul Joanne se réjouit de constater que, dans la presqu'île de Guérande, « malgré l'affluence croissante de leur clientèle estivale, les hôteliers sont restés fidèles depuis plus de quinze années à leurs prix de pension et à la variété d'un menu plantureux. La sardine fraîche, la crevette, les huîtres, palourdes et bigorneaux, etc., sont servis couramment comme hors-d'œuvre sur la table de la plupart des hôtels. Malgré l'absence de vignobles, presque aussi absolue que dans tout le reste de la Bretagne et dans la Normandie, le vin forme la boisson habituelle, sans supplément de prix. Les avantages d'un séjour sur cette côte sont particulièrement sensibles pour les baigneurs qui louent une villas ou une maisonnette, afin d'y vivre en famille ${ }^{71}$ ». Paul Gruyer indique que le coût de la vie est peu élevé à Quiberon en $1914^{72}$. Néanmoins, les petits prix pourraient faire penser à une pauvreté commerciale. La plupart des guides expliquent un approvisionnement à moindre coût grâce à un ravitaillement facile et abondant. Dans les années 1920, le Syndicat d'Initiative des Plages de Lannion avertit : "Beaucoup de baigneurs, s'imaginant trouver difficilement sur place ce dont ils ont besoin, ont l'habitude de faire expédier de Paris une grande partie de leurs provisions, ce qui pouvait avoir sa raison d'être au début. Il n'en est plus de même aujourd'hui, depuis que des maisons sérieuses et bien assorties se sont installées un peu partout et qu'elles livrent à domicile toutes les commandes à des prix excessivement raisonnables ${ }^{73}$."

Les stations balnéaires bretonnes qui se disent moins chères s'appuient sur la fidélisation d'une clientèle, loin de l'anonymat de la foule qui permettrait toutes les dérives. En 1936, le curé de Saint-Marc, plage de SaintNazaire, proclame la modestie de la station :

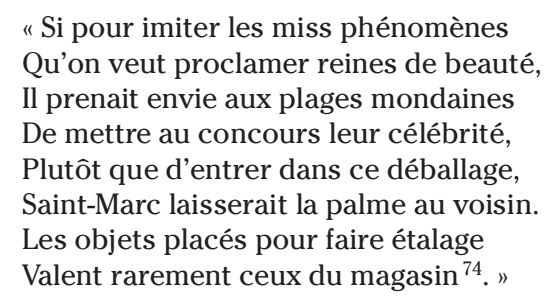

Si la venue de ces touristes ne réclame théoriquement pas de gros frais, ce qui expliquerait le prix modeste du séjour, en réalité équiper la station est nécessaire. Les coûts des travaux réalisés pour la station doivent être minorés. L'auteur du guide La Fare en 1903 indique que Port-Navalo devient une station balnéaire importante avec son poste de secours tout en restant " dans toute l'acceptation du mot un "petit trou pas cher" 75 ". Les petites

71. JoAnne, Paul, Itinéraire général de la France : la Loire, Paris, Hachette, 1900, p. 308.

72. GRuYER, Paul, Bretagne, Paris, Hachette, 1914, p. 383 et p. 457.

73. Lannion et ses plages, Éd. Syndicat d'Initiative des Plages de Lannion, sans date (1925), hors pagination.

74. Bulletin paroissial de Saint-Marc du 23 août 1936.

75. « Les petits trous pas chers »..., op. cit., p. 265. 
bourses ne souffriront pas de la présence de ce poste de secours. En 1921, le conseil municipal de La Turballe ne se déclare pas intéressé par la taxe de séjour, disponible pour moderniser et embellir la commune, car " les familles qui viennent passer la belle saison à La Turballe sont assez peu nombreuses et la plupart [d'entre elles] appartiennent à des familles de fonctionnaires qui recherchent plutôt des localités où n'existent pas d'impositions $^{76}$ ". L'action touristique doit savoir rester discrète pour que les touristes ne se doutent pas au cours de leur séjour de la rentabilisation des efforts consentis.

\section{Un séjour authentique}

Les touristes sont conscients que leur présence attise les convoitises. Il ne faut pas croire qu'avant les congés payés de 1936, ce ne sont que quelques rares et riches individus qui séjournent sur le bord de mer, notamment à partir de la fin du XIX ${ }^{\mathrm{e}}$ siècle. L'écart entre population sédentaire et population estivale à Pornichet est demeuré le même en 1900 et aujourd'hui : 1263 habitants contre 7000 à 10000 baigneurs en 1900, 10000 habitants contre 55000 touristes au début des années 2000, soit une multiplication par environ 6 entre l'hiver et l'été. Anne-Marie Gouriou retrouve à PerrosGuirec et à Trébeurden les mêmes écarts de 5 à 6 en 1932 et 1933 et, même au cours de la plus mauvaise saison des années 1930 à Perros-Guirec, la population durant l'été 1935 est multipliée par trois, soit plus de 8000 estivants ${ }^{77}$. La propagation des congés payés a été facilitée par la salarisation de la population active française après la Seconde Guerre mondiale; lorsque la loi des congés payés est votée, en juin 1936, la moitié de la population active travaille dans l'agriculture et n'est donc pas concernée. La station balnéaire bretonne n'échappe pas aux dérives touristiques. En 1905, Gustave Geffroy voit dans son hôtel de Concarneau « une mise en scène des villégiateurs installées dans les villes des pêcheurs " :

"La patronne de l'hôtel a gardé, sinon le costume du pays, du moins sa coiffe caractéristique, mais c'est du trompe-l'œil, comme les meublés bretons fabriqués à Paris, expédiés dans les petites villes armoricaines, chez les marchands d'antiquités. [...] Là-dedans, une réunion de toilettes féminines, blanches et roses, qui semblent jouer un rôle dans une comédie où l'on mènerait la vie de château, et non dîner dans une auberge proche des bateaux à sardines. C'est excessif, et l'on ne voit pas pourquoi l'habit noir, rouge, ou de la couleur que l'on voudra, n'est pas de rigueur à cette table d'hôte : c'est trop ou trop peu, trop snob ou trop négligé. [...] Mais les malheureux qui passent leur vie à Paris, dans des souliers vernis, du linge empesé, la tête encerclée par un chapeau dur, devraient avoir le droit, ici, de se libérer quelque peu du code pénal de la mode. Ce Concarneau est à fuir, s'il faut subir la loi qui sévit sur les plages normandes ${ }^{78}$. "

76. Délibération municipale de La Turballe, du 6 février 1921.

77. Gouriou, Anne-Marie, Le tourisme sur la Côte..., op. cit., p. 22.

78. GeFFroy, Gustave, La Bretagne, Paris, Hachette, 1905, p. 358-359. 
Afin d'éviter cette artificialité, des contre-modèles sont invoqués. Jusqu'au début de la concurrence estivale de la Côte d'Azur, les stations balnéaires normandes et les grandes stations thermales font figure de repoussoir. Léon Trebuchet conseille Belle-Île non " aux personnes qui recherchent les plaisirs des villes d'eaux, les distractions des casinos de bains de mer, mais à celles qui désirent le calme et pour lesquelles les beautés de la nature ont quelque attrait. Belle-Isle ne ressemble, sous aucun rapport, aux plages de la Normandie, vers lesquelles les élégants accourent de tous côtés. Là, point de ces toilettes excentriques comme à Dieppe, Trouville, Dinard ou Pornichet. La vie s'y écoule calme et, si l'on rencontre quelques baigneurs aux plages Hallan ou Ramonette, au port Guen ou au port Fouquet, ils ne s'y rendent que pour leur plaisir personnel et non pour attirer les regards ${ }^{79}$ ". Le séjour balnéaire en Bretagne se déroule sans contraintes sociales. Malgré de nombreux discours vantant les animations, la station balnéaire est un lieu de calme, une rupture avec le quotidien et un retour à la nature. Pour Léon Warenghem en 1899, le séjour à Trébeurden ravira celui « qui vient au bord de la mer dans le but d'y éprouver les nobles jouissances que procurent la vue et l'étendue de la nature. Il convient aussi aux enfants, aux familles qui recherchent un endroit tranquille et pittoresque, loin des agitations de la vie mondaine, aux rentiers qui veulent trouver à la fois la santé, le repos et la vie bon marché ${ }^{80}$ ". L'Hôtel du Chalet, à Saint-Brévin-l'Océan, énumère dans sa publicité, en 1931, son confort, son parc merveilleux et sa proximité avec la mer, toutes ces qualités qui "vous assurent du plus reposant et du plus agréable séjour ${ }^{81}$ ".

Les touristes recherchent l'intemporalité dans les paysages et chez les populations locales. En se promenant de Dinan à Saint-Malo, " nous voulons saluer avec vous ces rochers géants vieux comme le monde, ces coulées ombreuses que les Faunes et les Sylvains semblent habiter encore, ces collines ininterrompues, couronnées ici de futaies, de blonds épis ondoyant sous les brises de mer, là d'humbles cabanes de pêcheurs, plus loin de villas éclatantes environnées de jardins embaumés, gracieuses comme de jeunes reines au milieu de leurs cours ${ }^{82}$ ". En 1879, " les mœurs, les costumes, les habitants, offrent une étude d'autant plus rare que les moyens de communication si rapides et si multipliés, ont enlevé aux provinces leur couleur et leur originalité en répandant partout une teinte d'uniformité et parfois de monotonie qui n'a pas encore atteint la Basse-Bretagne ${ }^{83}$ ". Néanmoins, paysage comme habitants évoluent. L'influence des baigneurs

79. TRÉBUCHET, Léon, Les Étapes d'un touriste..., op. cit., p. 121.

80. WARENGHEM, Léon, En Bretagne; Trébeurden, ses îles, ses grèves, son climat, ses légendes, Lannion, Imprimerie Anger, 1899, p. 66.

81. Dardelin, Henri, Saint-Brevin-les-Pins, Paris, Le Livre d'histoire-Lorisse, coll. "Monographies des villes et villages de France ", 2002 [1 ${ }^{\text {re }}$ éd. 1931], p. 127.

82. BAzouge, J., Guide du voyageur dans la ville de Dinan et ses environs, Dinan, Librairie Bazouge, sans date (années 1860?), p. 236.

83. LE GAL, E., Guide du touriste; Rhuys, Locmariaquer, Gavr-inis, questions archéologiques, Vannes, 1879, p. 32. 
est alors considérée comme préjudiciable à la Bretagne. A. Gahéry prévient dans les années 1900 les jeunes filles bretonnes qu' "en cédant à un courant fâcheux, en vous emparisiennant, vous perdriez le meilleur de vousmêmes, vous perdriez tout ce que vous espériez gagner ${ }^{84}$ „. En 1930, André Chevrillon dénonce l'automobile dans les chemins, les grands cars au-dessus des grèves où se trouvaient jadis les brûleurs de goémons, les rangs de villas enveloppant les villages indigènes, l'auberge muée en hôtel des baigneurs : "Les granits même, qu'on croyait éternels, sont attaqués ${ }^{85}$. " La population bretonne est appelée à défendre ses traditions qui apparaissent alors plus touristiques qu'authentiques. D'autres auteurs s'éloignent de la foule pour retrouver la Bretagne qu'ils attendent :

"Les touristes qui se ruent à Penmarc'h devraient savoir que Loctudy, avec ses anses, sa mer violette, son phare rouge, ses barques et ses cargos, est l'un des plus pittoresques et des plus doux coins de Bretagne. Une lumière blonde baigne la côte dentelée et l'île de Tudy. Un caboteur part pour Cardiff. Des femmes travaillent, laborieuses, sur les quais. Des marins flânent, dont la franche figure est gallique Les bois de pins ombragent les plages de sable fin. Voici le Paradis de la Cornouaille du Sud, le pays des dentelles de fées, dont toutes les femmes font emplette cependant que les hommes, attablés au marbre du "débit de boissons", dégustent un cidre de Plomelin, qui est l'ambroisie de la terre ${ }^{86}$."

La Bretagne n'échappe pas aux limites de l'authenticité, née du jugement de la personne extérieure et non d'une réalité intrinsèque, mais son potentiel semble régulièrement renouveler cette légitimité.

La station balnéaire bretonne est le produit complexe de l'aménagement de l'espace. Elle est à la fois un espace foncier, parcellisé et quadrillé de voies, et un espace de vie, occupé par une population permanente ou de passage. Chaque station balnéaire a donc ses caractéristiques, sur lesquelles elle s'appuie comme des atouts. Étudier la typologie des stations balnéaires montre néanmoins que le phénomène balnéaire n'a rien d'unique en Bretagne. Le territoire est utilisé de la même manière que sur d'autres littoraux français. Pourtant, même quand chaque station balnéaire a œuvré pour montrer sa particularité, la Bretagne a gardé une image commune qui la différencie des autres. Cette image " provinciale " doit beaucoup au tourisme né des échanges renouvelés chaque année. En multipliant les sites, souvent délaissés et réemployés, il a diffusé l'image de la petite station balnéaire bretonne tandis que les populations locales actives participaient à l'élaboration d'un tourisme à l'échelle humaine. La longueur du trait de côte a permis à la Bretagne de se créer une image touristique, se nourris-

84. Gahéry, A., Port-Louis; Casino, plage, environs, sans date (années 1900), non paginé.

85. Chevrillon, André, La Bretagne, 1930, Paris, Lévy et Neurdein, p. 2.

86. Le Povre Moyne, Jehan, À travers la Bretagne, Le Havre, Journal Le Petit Havre, 1929, p. 47 . 
sant des a priori des villégiateurs et se démarquant ainsi des autres régions concurrentes au littoral plus restreint.

Il est possible que les stations balnéaires connaissent à l'avenir un certain développement. Le passage à la ville est un trait commun à de nombreuses stations, y compris celles créées ex nihilo comme sur la côte du Languedoc-Roussillon. Les stations balnéaires sont investies non plus seulement à la belle saison par les baigneurs mais également à l'année par des résidents permanents. Ce processus bouleverse l'équilibre économique et démographique des régions concernées, avec une montée en puissance de ces entités littorales par rapport à un hinterland affaibli, à tout point de vue. La diversification de l'économie locale est largement une conséquence de la sédentarisation des villégiateurs, les résidences secondaires devenant résidences principales. En vivant à l'année dans ces stations, ils soutiennent l'économie de la station et s'impliquent dans la vie locale, politique ou associative. Ce faisant, ces résidants réalisent la mutation de la station balnéaire en une ville à part entière. Les nouveaux modèles de la ville se trouveront peut-être dans les stations balnéaires.

\section{RÉSUMÉ}

Les pratiques balnéaires ont plus de deux siècles en Bretagne. Elles ont fortement marqué la région, tant dans la nouvelle utilisation du territoire que dans l'identité et l'imaginaire de cet espace. Une typologie complète des stations balnéaires bretonnes montre qu'elles peuvent se rapporter à trois modèles différents : la station créée ex nihilo, la station avec son quartier de la plage, la station avec plusieurs quartiers balnéaires. L'évolution de chacune est diverse et prouve les interrelations entre différentes communes souvent considérées comme concurrentes. Néanmoins, toutes ces différences peuvent se rejoindre ou s'effacer pour former un même discours touristique autour de la Bretagne. Si la station balnéaire vante ses atouts, la Bretagne a aussi ses caractéristiques, reprises au niveau local. L'action touristique influence fortement ces discours qui mettent en avant l'absence des promoteurs, la maritimité, un tourisme modeste, la vie peu chère et l'authenticité. La station balnéaire bretonne est l'héritage complexe de deux siècles d'histoire balnéaire.

\section{ABSTRACT}

Seaside stays began two century ago in Brittany. It led the country to a new use of the littoral and a new identity. Seaside resort created ex nihilo or with its seaside area or with many seaside suburbs. Each evolved and the development proved interactions between these cities often rivals. However, commons qualities appeared in tourists advertisements in Brittany: no promoters, maritimity, cheap way of life and authentic stays. Seaside resort of Brittany is the complex result of maritime history. 